\title{
Adaptive Side-by-side Social Robot Navigation to Approach and Interact with People.
}

\author{
Ely Repiso · Anais Garrell • Alberto Sanfeliu
}

Received: date / Accepted: date

\begin{abstract}
This paper presents a new framework for how autonomous social robots approach and accompany people in urban environments. The method discussed allows the robot to accompany a person and approach to other one, by adapting its own navigation in anticipation of future interactions with other people or contact with static obstacles. The contributions of the paper are manifold: firstly, we extended the Social Force model and the Anticipative Kinodynamic Planner [1] to the case of an adaptive side-by-side navigation; secondly, we enhance side-by-side navigation with an approaching task and a final positioning that allows the robot to interact with both people; and finally, we use findings from experiments of real-life observations of people walking in pairs to define the parameters of the human-robot interaction in our case of adaptive sideby-side. The method was validated by a large set of simulations; we also conducted real-life experiments with our robot, Tibi, to validate the framework described for the interaction process. In addition, we carried out various surveys and user studies to indicate the social acceptability of the robots performance of the accompanying, approaching and positioning tasks.
\end{abstract}

Keywords Human-Robot companion side-by-side, Human-Robot approaching, Robot Navigation,

Work supported by the Spanish Ministry of Science and Innovation under project ColRobTransp (DPI2016-78957-R AEI/FEDER EU) and by the Spanish State Research Agency through the Maria de Maeztu Seal of Excellence to IRI (MDM-2016-0656). Ely Repiso is also suported by Spanish Ministry of Science and Innovation under a FPI-grant (BES2014-067713)

The authors are with the Institut de Robòtica i Informàtica Industrial (CSIC-UPC). Llorens Artigas 4-6, 08028 Barcelona, Spain. \{erepiso, agarrell, sanfeliu\}@iri.upc.edu
Human-Robot Interaction, Human-Robot Collaboration.

\section{Introduction}

In order to share our environment with robots in a comfortable manner, we need to endow them with the cognitive functions that will enable them to assist and collaborate with people as they go about their daily tasks [2-4]. These collaborations will improve our quality of live by compensating our shortcomings to perform certain tasks. As for example: Companion robots can allow elder people to fend for themselves or complement the people abilities to do a better work. Then, society is evolving to obtain social robots to help people and this evolution may change the society to have a more independent elder population. There is a mutual shaping between this new technology and society, as the robots and humans will share spaces and tasks in cities. Therefore they will need to adapt and change the way that robots and people will interact and solve tasks.

Among the skills that social robots might require include: guiding people [5-7], following pedestrians [8, $9]$, accompanying people [10-12], learning how to approach people and develop original ways of reaching them $[13,14]$, or understanding human behaviour $[15$, 16]. If robots are to be integrated into society in order to assist people in their daily tasks, they first must be able to accompany and approach us; accompaniment is essential for performing tasks jointly, if robots are to be accepted in society. These robot companion and approaching skills may be useful in tasks like: shopping assistants, [17], telepresence robots [18], social companions for the elderly or infirm, [19,11], or wheelchair au- 


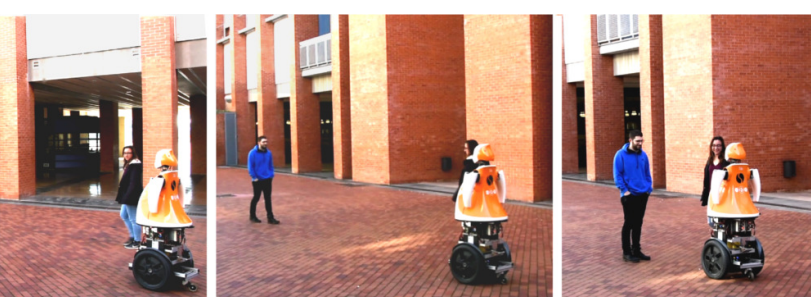

Fig. 1 Real-life experiments of the user study: Left: Tibi Robot accompanies a person in an urban environment. Center: The pair robot-human, approaches a pedestrian in the surroundings. Right: The three members share the personal space while have a conversation.

tonomous systems that are able to walk side-by-side with their care givers [20-22], joining a static or dynamic group in conversation, [23], or simply requesting directions or other information [24].

This paper presents a new framework where a social robot is able to accompany a person, attempt to approach a known pedestrian moving in the same environment, and interact with both individuals, Fig. 1.

Therefore, our method requires that the robot accomplish three essential tasks: (i) accompany a human in a way perceived as comfortable; (ii) determine the best way of approaching a moving person while accompanying another; and (iii) be able to position itself appropriately during the final static interaction stage.

The first task, that of walking side-by-side or accompanying another person, is instinctive for humans, but this is not an easy endeavour for robots. Pedestrians walking in groups are able to understand the spatial situation and adjust their structure accordingly. Normally, people walk in side-by-side configurations, but they may break this formation on occasion, in order to cross narrow passages, avoid obstacles, and generally facilitate the navigation of other pedestrians in the environment. To acquire this behaviour, social robots need to be able to not only predict the peoples behaviour within the immediate surroundings, but also infer the motion of those being accompanied.

The second task, that of approaching a person in the environment, is of great importance, seeing as people constantly interact with one another while walking, to get directions, ask a stranger to take a photograph, etc. In this task, the most challenging aspects are recognizing the nature of the situation, determining the best way of approaching a human while accompanying another, and effectively predicting the motion of other pedestrians in the area.

The third task is that of having the robot place itself within the social group in a way that fosters interaction between all group members. At that juncture, the robot has to understand the situation and be able to position itself in order to face both people and be part of the conversation.

Additionally, the more complex dynamics of this scenario requires that the robot be equipped with additional perception skills, such as ascertaining each persons orientation, and predicting the future positioning of the group. The challenge is further complicated by the fact that the target person might be momentarily hidden from view by other people or obstacles, forcing the robot to respond appropriately to myriad uncertainties.

Later sections of this paper review, the related work in Section 2. Section 3 presents the companion framework in depth, while the approaching method itself is presented in Section 4. Section 5 describes the learning procedure used in order to determine the parameters for the interaction between the robot and the accompanied person. The performance metrics used to evaluate the tasks are given in Section 6. In Section 7, we share the results of the synthetic experiments. The algorithm is tested extensively in real-life experiments in Section 8, where also the robot platforms and the experimental environments are described. A User Study of the complete task appears in Section 9. Furthermore, the discussion is included in Section 10. Finally, the conclusions and future work are given in Section 11.

\section{Related Work}

As mentioned above, our ultimate objective is to make robots capable of accompanying people while approaching a dynamic target (in this instance, a known pedestrian) and successfully interaction with both people. To perform this complex task, the robot needs to complete various feats: perform social autonomous navigation; accompany a person; and approach a pedestrian, while conforming to social conventions.

\subsection{Autonomous Navigation}

Autonomous navigation is a required skill in social robotics, given that robots are designed to share pedestrian spaces with people and assist them $[25,26]$.

Early works on navigation did not take into account the standard conventions of social navigation between people. In [27], the researchers developed an optimised a navigation method based on optimal time or distance to arrive at the relevant target. Foka et al. [28] implemented a planner based on POMDPs which uses motion prediction in order to analyse the dynamic obstacles 
obstructing the path between the robot and its desired destination.

Other authors have developed more complex navigation behaviours to enable robots to handle crowded environments. [29] implemented maximum entropy inverse Reinforcement learning to develop robot behaviours that mimic human behaviours. Trautman et al. [30] used a navigation algorithm based on a probabilistic predictive model of cooperative collision avoidance, which allowed the robot to navigate in densely crowded areas.

In addition, several navigation approaches set out to first determine aspects of human navigation behaviours. Kuderer et al. [31] employed a learning method to predict pedestrian movements and applied the model to produce a robotic behaviour that would be socially compliant therewith. Luber et al. [32] presented an unsupervised learning method to produce a set of relative motion prototypes which enabled the robot to compute a dynamic cost map, and to plan socially acceptable avoidance manoeuvres. These trajectories optimised travel time, path lengths, and social comfort.

Our work applies the Anticipative Kino-dynamic planner [1], a means which allows the robot to navigate within challenging crowded environments finding the optimal path. One approach that bore some resemblance to this type of planner is that developed by Sisbot et al. [33], which carefully considered the social rules between humans to improve the robot's approach, paying close attention to field of vision and accessibility to humans.

\subsection{Human-Robot Accompaniment}

Walking side-by-side is a collaborative task in which the human-robot pair shares a common goal. This demands that the robot know the destination and is able to adjust its navigation in accordance with the movements of its partner. The challenge here lies in the fact that the robot must predict and learn the persons behaviour, anticipate his or her movements, and respond appropriately. Because this aspect is essential, we endeavoured to review previous works that addressed the task of robot accompaniment.

As a starting point, some researchers developed strategies for robots following and guiding people. [9] used BIRON to implement a context-aware following behaviour, which alternated between three basic models of following behaviour: direction-following, path-following, and parallel-following.

Later, others developed hybrid approaches that combined following, guiding, and accompanying behaviours.
Ohya et al. [34] used a robot capable of following or accompanying a person by predicting the person's next position and advancing accordingly, so as to place itself by his or her side. Pang et al. [18] developed a multimodal person follower that allowed a telepresence robot to guide, follow, and walk side-by-side with a person.

Moreover, [35] introduced a following behaviour for a robot that could be perceived by a non-expert as merely following someone, or as a guiding companion. Their system involved an anticipatory behaviour that was able to follow the human from different positions: from the front, from the rear, and from the side.

Recently, researchers have developed more complex strategies in their work on social robots [36]. Morales et al. $[37,12]$ proposed a model of people walking sideby-side which could predict the partner's future position, and subsequently generate a plan for the robot's next position. Furthermore, [38] and more recently [39] did a side-by-side method inferring the final goal of the person and also recorded a database of people walking in a fixed side-by-side formation that is different from our database, included in Section 5, which includes also situations of an adaptive side-by-side companion behaviour. Kuderer et al. [40] explored the possibility of confronting the problem by using reinforcement learning methods in order to teach a tele-operated robot to navigate in a cooperative way while avoiding collisions with obstacles.

The most complex approaches developed to date have been designed for use on wheelchairs, a social necessity. Prassler et al. [20] implemented a method of accompaniment for a wheelchair, designing a model of collision avoidance based on velocity that incorporates a linear prediction of collision velocities. Kobayashi et al. [21] used a visual-laser tracking technique to carry out a side-by-side companion task between a wheelchair and a caregiver, demonstrating the same effect within the context of visiting a museum. Finally, Suzuki et al. [22] proposed a wheelchair system that navigates in a formation which renders a more natural communication between the user and the caregiver.

While previous studies only discuss the challenge of navigating around the person in a fixed side-by-side formation, our algorithm allows for a more dynamic positioning around the human partner. This is to say, the method allows the robot to position itself at front, at lateral and at back of the person who accompanies depending on the situation. Then, if no obstacle interferes with the group's path, the robot accompanies the person in a side-by-side, but if any obstacle interferes with the group's path, the robot changes its position around the person to avoid them. One of the most interesting cases is when other people or obstacles interferes 
with the robot's path for a long time. Then, the robot surrounded the person who accompanies and position itself in the other side, where it had free space. Furthermore, our method tries to always select as best path one of the possible paths that allow the accompaniment in side-by-side, as long as the person does not force the robot to go through another path that has less space. Using this method, the robot facilitates the group's navigation within dynamic environments and eases the group's burden of avoiding collisions with other people. Also, the robot facilitates the navigation of other people in the environment avoiding to interfere in their path. Resulting in a more similar accompaniment to human behavior when we accompany other people.

Another innovation that sets our approach apart from others is that our method is able to render a real-time prediction of the dynamic movements of the partner, as well as that of other people, in a horizon time. This kind of prediction performed within a determined time window allows the robot to anticipate people's navigation and react accordingly.

Furthermore, our method allows that the robot and the person who accompanies start separately. When the robot identifies the companion, it approaches to the companion side. Also, the robot selects the side of the person with more free space. Now we consider that for the person it is indifferent the side where the robot accompanies that one.

\subsection{Human-Robot Proxemics Rules}

Proxemics rules were first introduced by Hall [41]. Some researches agree that the proxemics rules between a person and a robot should be similar to the walking rules among people [25] and we have followed this idea in this work.

In [42], researchers studied how personality affects personal space. They defined four personality aspects: proactiveness, social reluctance, timidity, and nervousness; and concluded that proactive subjects typically get closer to the robot than do people who are less confident with or around the robot. Moreover, negative attitudes towards robots and direct gazes between a person and a robot usually implies to increase the distance between them. Syrdal et al. [43] studied the distances between robots and people within different situations of interaction: verbal, physical, and no interaction. One of the outcomes of their research was that females often allow the robot to come closer to them than do their male counterparts. Some studies [44] also shown the preferences of people with respect to the approaching side, for example, right-handed people prefer to be approached from their right side.
More closely aligned with our research objectives, Satake et al.[14] endeavour to have the robot approach a person walking in a shopping mall and announce sales or news that might be of interest.

In contrast to our work, previous researchers did not explore different cases and situations of approximation. The present study also stands out in that it addresses not only the robots navigation, but also endeavours to allow the robot to accompany a person familiar with the target person, while respecting social norms.

\subsection{Robots Approaching People}

The Human-Robot approach is an important collaborative task that takes place between humans and robots in order to generate interaction; central to this task is the ability to recognise and predict the intentions of the other partys movements.

In the past, researchers have used different control strategies for approaching a moving target by either pursuing it, or trying to intercept it [45-47]. In [47], the researchers employed a wheeled robot control technique that enabled the robot to intercept a moving target from a particular approach angle. They use a navigation technique that maintain steady contact with the target from a certain geometrical position until ultimately intercepting it.

Fajen et al. [48] presented different control strategies for approaching a moving target. Narayanan et al. [23] used a task-based control law to enable the robot to meet two standing persons and interact with them, by carefully considering their respective positions and orientations, and use that knowledge to calculate an optimal meeting point.

Other researchers $[49,24]$ studied human social behaviours in order to yield better results. [49] recorded the different trajectories that people took when approaching other persons. They later used those trajectories to develop a reward function that allows the robot to follow human-like trajectories. Kato et al. [24] developed a robot behaviour model for the interaction between shopping mall staff and the customers. In [50], the authors used proxemics rules to define the approaching distance to the target person for teaching robots proactive behaviour.

Finally, Carton et al. [13,51] presented a method which uses Bezier curves to incorporate socio-contextual human features into the robot's planned path of approach.

In contrast to the previous approaches, our work employs a prediction module based on the social force model, which includes human-like behaviours for navigating within dynamic environments, and for mapping 
out the best path for the robot to take towards a moving destination. We are also able to compute the best meeting point between parties by considering the status of the group, the state of the target person, and the target person's future position. Our method also resulted in a more accurate determination of a specific meeting point than that yielded by other methods, which typically do not consider people's future movements nor imagine the myriad possible paths towards intercepting the target person. Then, our method allows the robot to predicts the best meeting point in situations where the approached person is stop or walking in any direction within the environment, either towards the group or away from it. Also, these paths and the final meeting point take into account static and dynamic obstacles of the environment. Also, at the same time the robot was adapting to other person movements (the companion) that can modify the path of the group. Furthermore, in the final stage where the group is near of the approached person, our framework is based on human behaviour, wherein people who are standing up (either static or dynamic) arrange themselves in geometric formations in order to participate in a conversation.

In addition has to be mentioned that none of the previous works implemented our three stages at the same time: accompanying, approaching and positioning. Also, we search for literature that did it, but we could not find any work about it.

\section{Adaptive Human-Robot Accompaniment Navigation}

In this section, we present the adaptive Human-Robot accompaniment navigation, which makes robot capable of navigating in an adaptive side-by-side formation while accompanying a person in dynamic environments. The objective of this accompaniment task is that the group robot-person, denominated by $g$, reach a destination goal $D_{n}{ }^{g}$, in an environment with static obstacles $o$, and with dynamic obstacles due to other people $p$ that are moving (in this work we only consider the moving people as dynamic obstacles). However, it is important to understand that is the person of the group who selects its own destination goal $D_{n}{ }^{g}$ at each instant of time, which is not known by the robot $r$, and that we can only control the robot. This means that the robot has always to predict the intention of the person to know the destination goal. Also, the robot has to do a dynamic accompaniment to avoid the static obstacles and the dynamic obstacles by predicting the intention of the other moving persons that can cross the group $g$.

To achieve this type of navigation, the robot has to evaluate all possible paths until the final destination and select the best one taking into account: minimum path distance, minimum difference of orientation until the final destination, minimum effort to avoid obstacles or pedestrians, and the best formation with the accompanied person. Usually, the pair robot-human will navigate keeping the side-by-side formation, but sometimes they have to modify it to pass through a narrow passage or to avoid groups of people.

The adaptive Human-Robot accompaniment navigation method described below, is composed by several elements: inferring the person destination goal to be used as group goal; planning the path to reach the goal avoiding the static and dynamic obstacles; predicting the pedestrian movements to avoid the collision with them; maintaining the side-by-side formation while is navigating; and controlling the robot to accomplish with the accompaniment task. For inferring the person destination goal we have used the method BHMIP [52] and for the planning in crowded environments we are based on the Anticipative Kino-dynamic Planner (AKP) [1], which allows the robot to navigate in challenging crowded environments while performs a social acceptable navigation. In this section we will explain the new changes in the evaluation of the paths due to the side-by-side formation and the new formulation of the Extended Social Force model to do the adaptive accompaniment navigation to achieve the side-by-side navigation.

This section is divided in three parts, which represents the three steps to be solved: (i) to infer the person destination, to plan all the paths using the AKP and the Extended Social Force model, (ii) to evaluate all the planned paths using an additional costs function due to the side-by-side formation, and (iii) to extend the social force model for the accompaniment task.

3.1 Stage 1: Infering the person destination, planning all the possible paths with AKP and formulating the Social Force Model for the accompaniment task

First of all, the robot needs to know the final destination of the person who is being accompanied, who can change its destination at any time. To obtain this information, we have used the Bayesian Human Motion Intentionality Predictior (BHMIP) method [52], which is a geometric-based long term prediction method that uses a Bayesian classifier to compute the best prediction to a given destination position. The BHMIP method assumes that the set of possible destinations inside the dynamic environment $D=\left\{D_{1}, D_{2}, \ldots, D_{n}, \ldots, D_{m}\right\}$ are known. These destinations are usual points where people go, as entrances, exits or work places. In this section 
we will assume that the destination goal for the group $g$ is $D_{n}^{\text {goal }}$.

Once the robot knows the final destination, it has to compute all the possible local paths using the AKP local planner. This local planner uses the Social Force Model (SFM) to analyze which trajectories are correct and to analyze all paths at each control time, considering the static obstacles and the predicted location of the dynamic obstacles (moving pedestrians).

Let us first formulate the accompaniment task to reach a destination goal using the SFM considering the group $g$, see Eq. 1.

$\mathbf{F}_{g}=\alpha \mathbf{f}_{g, d}^{\text {goal }}\left(D_{n}{ }^{\text {goal }}\right)+\left(\gamma \mathbf{F}_{g}^{\text {ped }}+\delta \mathbf{F}_{g}^{\text {obs }}\right)$

Where $\alpha, \gamma$ and $\delta$ parameters are the weights of the respective forces, and $\mathbf{F}_{g}$ is the resultant force of the group, $\mathbf{f}_{g, d}^{\text {goal }}\left(D_{n}{ }^{\text {goal }}\right)$ is the attractive force until the final destination of the group, $\mathbf{F}_{g}^{p e d}$ and $\mathbf{F}_{g}^{\text {obs }}$ are the repulsive forces from pedestrians and obstacles respect to the group.

These parameters were learned using a Markov Chain Monte Carlo Metropolis-Hastings method ( MCMC$\mathrm{MH}$ ) over data extracted from simulations of the planning task, and then were validated in the real life experiments. For more information about this procedure the reader is referred to [53].

The attractive force until the final destination is defined in Eq. 2. This attractive force assumes that the group $g$ tries to adapt its velocity within a relaxation time $k^{-1}$, but taking into account at the same time to arrive to the final destination inferred by the BHMPI.

$\mathbf{f}_{g, d}^{\text {goal }}=k\left(\mathbf{v}_{g}^{0}\left(D_{n}^{\text {goal }}\right)-\mathbf{v}_{g}\right)$

$\mathbf{v}_{g}^{0}\left(D_{n}{ }^{\text {goal }}\right)$ is the desired velocity vector to reach the final destination $D_{n}{ }^{\text {goal }}$, which is inferred from all the destinations of the environment using BHMIP, and $\mathbf{v}_{g}$ is the current velocity of the group. This force pushes the group to their final destination.

To avoid pedestrians, we define the following repulsive force:

$\mathbf{F}_{g}^{p e d}=\sum_{j \in P} \mathbf{f}_{g, j}^{i n t}$

$\mathbf{f}_{g, j}^{i n t}=A_{g p} e^{\left(d_{g p}-d_{g, j}\right) / B_{g p}} w\left(\varphi_{g, j}, \lambda_{g p}\right)$

$A_{g p}, B_{g p}, \lambda_{g p}$ and $d_{g p}$ are the parameters of the group-person interaction, in Section 5. $A_{g p}$ and $B_{g p}$ denote respectively the strength and range of interaction force, $d_{g p}$ is the sum of the radii of a pedestrian and an entity and $d_{g, j}=r_{j}-r_{g}$. We define how these parameters were obtained in the case of the interaction force between the robot and the accompanied person. For the robot and all the other people of the environment were learned these parameters in a previous [53] work using the same procedure. Here, these interactions could be between robot-person, person-person and group-person. In the case of the group we use the value of the parameters of the robot-person interaction, since our model use the larger component of the group, in our case the robot, to be able to pass through narrow passages in one-by-one formation.

Given the limited human's field of view, influences might not be isotropic. This is formally expressed by scaling the interaction forces with an anisotropic factor depending on $\varphi_{g, j}$.

$w\left(\varphi_{g, j}\right)=\left(\lambda_{g p}+\left(1-\lambda_{g p}\right) \frac{1+\cos \left(\varphi_{g, j}\right)}{2}\right)$

$\lambda_{g p}$ defines the strength of the anisotropic factor.

$\cos \left(\varphi_{g, j}\right)=-\boldsymbol{n}_{g, j} \cdot \boldsymbol{e}_{g}$

The term $\boldsymbol{n}_{g, j}$ is the normalized vector pointing from the center of the group to person $p_{j}$ which describes the direction of the force, then $\varphi_{g, j}$ is the angle which describes the group's motion.

Finally, the repulsive force respect to the obstacles in the environment is defined by:

$\mathbf{F}_{g}^{o b s}=\sum_{o \in O} \mathbf{f}_{g, o}^{i n t}$

$\mathbf{f}_{g, o}^{\text {int }}$ is obtained equivalent to Eq. 4. The main difference is that now the interaction is with an object instead of a person.

These repulsive forces are the summation of all the repulsive forces between the group and all pedestrians and obstacles.

3.2 Stage 2: Evaluation of the planned paths using a new cost function for the accompaniment task

At each time $t$, the robot computes all possible paths for the group $g$ using the AKP, but only one will be the optimal one. The robot should evaluate and select the best one using a cost function that takes into account the different constraints (obstacles) and optimization criteria. In [54], there were defined a cost function that included the minimum path distance, minimum difference of orientation until the final destination, minimum effort to avoid obstacles or pedestrians. Because we have 
to accomplish a side-by-side formation with the accompanied person we have also to define a new cost function to add to the previous ones. The equation of this cost function is defined in Eq. 13.

Let us define the accompaniment cost function. This cost function has to take into account the distance between the robot and the accompanied person and the angle between them to obtain a side-by-side formation. Fig. 2 shows the geometrical parameters and variables to be taken into account.

$R_{g}$ is the radius of the circle containing: the robot (with radius $R_{r}$ ), the person (with radius $R_{p}$ ), and the minimum distance between the center of the position of the person and the center of the position of robot, $2 R_{i}$, where $R_{i} \geq 0.75 \mathrm{~m}$. The lowest value of $R_{i}=0.75$ $\mathrm{m}$ is obtained by tacking into account the radius of our robot, $0.5 \mathrm{~m}$, and the necessity of some free space between the robot and the person to allow some error tolerance (we have set up $0.25 \mathrm{~m}$ ). Then, the distance between the central positions of the robot and the accompanied person is $2 R_{i}=1.5 \mathrm{~m}$, this allows a real free space between them within the range of $[0.25-0.7]$ meters. These distances were chosen taking into account the radius of our robot, the standard person radius, and a previous work [7]. Furthermore, the following two equations must hold for $R_{i}$ and $R_{g}$.

$R_{i} \geq\left(R_{r}+R_{p}\right) / 2$

$R_{g}=R_{i}+\max \left(R_{r}, R_{p}\right)$

To define a cost function, we have to consider the distance, $2 R_{i}$, and the best angle between them, $\theta$. These two parameters allow the side-by-side navigation of the group while avoiding static and dynamic obstacles. To obtain the best angle, we compute the minimum collision distance $d_{o}$, Eq. 10, between the center of the pair and the nearest obstacle. Using the collision distance, $d_{w}$ is obtained which is the shortest distance between the center of the robot's position and the direction of movement of the group. This distance has a maximum value of $R_{i}$ when the group is in side-by-side formation. Then, $\theta$ can be obtained using Eq. 12. To calculate all of the collisions over the paths we use a predefined map and laser scans.

$$
d_{o}(t)>d_{w}(t)+\max \left(R_{r}, R_{p}\right)
$$

$d_{w}(t)=R_{i} \sin (\pi-\theta(t))$

$\theta(t)=\pi-\arcsin \left(\frac{d_{w}(t)}{R_{i}}\right)$, for $\theta(t) \in\left(-\frac{\pi}{2}, \frac{\pi}{2}\right]$

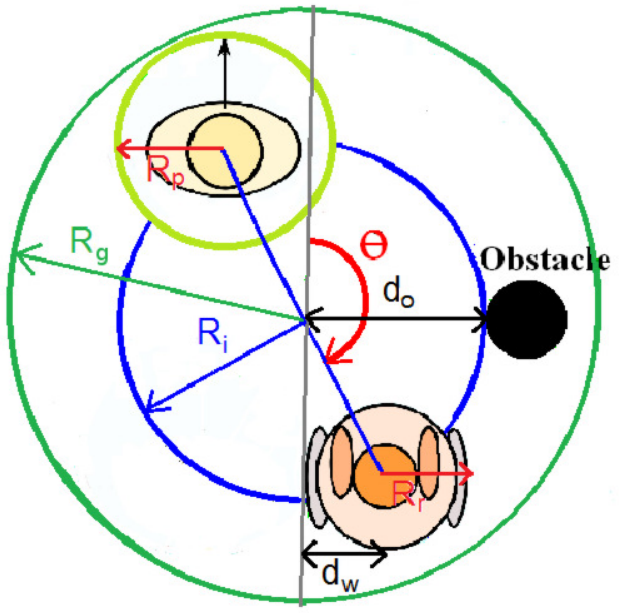

Fig. 2 Group positioning. Computation of the HumanRobot formation to avoid an obstacle while the robot accompanies the person.

Finally, the new cost $J_{c}$, denominated companion cost, is defined as follows:

$$
J_{c}(S)=\eta\left\|\theta(t)-\theta^{0}\right\|^{2}
$$

Where $S$ is the state of any entity: the robot, the group or pedestrians. The state includes position, velocity and time, and additionally orientation for the robot or group, see [1] for information about the states. $\theta^{0}$ is the best angle of companion, in our case $90^{\circ}$. Then, the minimum cost is obtained when the robot accompanies the person in a side-by-side formation, and this cost increases when the robot has to break this formation to avoid obstacles. We want that the robot always selects the path where the group can walk more time in a side-by-side formation. The graph of the companion cost can be seeing in [55]

This new companion cost, $J_{c}$, is added to the cost functions defined in the AKP method, obtaining a new cost function $\mathbf{J}\left(S, s_{\text {goal }}, U\right)$ with all the associated costs:

$$
\mathbf{J}\left(S, s_{\text {goal }}, U\right)=\left[J_{d}, J_{o r}, J_{r}, J_{p}, J_{o}, J_{c}\right]
$$

Please refer to [54] to know the details of the other cost functions. The computation of this cost function requires three steps. First, each individual cost function is computed. Second, in order to avoid the scaling effect of weighted-sum method, each cost function is normalized to $(-1,1)$, according to the equation:

$\bar{J}_{i}(\mathbf{X})=\operatorname{erf}\left(\frac{x-\mu_{x}}{\sigma_{x}}\right)$ 
The variables $\mu_{x}$ and $\sigma_{x}$ are estimated after the calculation of all RRT's possible planning paths. Finally, third, a projection via weighted sum $J: R^{I} \rightarrow R$ is obtained giving the weighted cost formula (see [54] for additional explanation):

$J\left(S, s_{\text {goal }}, U\right)=\sum_{i} w_{i} \bar{J}_{i}\left(S, s_{\text {goal }}, U\right)$.

The path with the minimum cost function, named as $J\left(S, s_{\text {goal }}, U\right)$, is selected as the best path among the possibles paths to the destination goal at each control interval.

3.3 Stage 3: Extension of the social force model for the companion task

In the previous subsection, we have incorporated an accompaniment cost function for the selection of the best path. Now, we include a new attractive force in the Social Force Model that takes into account the accompaniment factor to control the navigation of the robot. This new force tries to maintain together the group formed by the robot and the person while the group is navigating toward the destination goal. The resulting new force $\mathbf{F}_{r}$ is shown in Eq. 17. Then, the resultant force to control the robot movement has two attractive forces, one, $\mathbf{f}_{r, d}^{\text {goal }}\left(D_{n}{ }^{\text {goal }}\right)$, that pushes the robot to the destination goal inferred by motion intention of the accompanied person, and the second one, $\mathbf{f}_{r, p}^{\text {goal }}\left(D_{n}{ }^{f}\right)$, that pushes the robot to maintain the adaptive side-byside formation with the accompanied person. Moreover there are two repulsive forces analog to the repulsive forces for the group in Eq. 1 but now for the robot, one is the repulsive force due to the static obstacles of the environment $\mathbf{F}_{r}^{o b s}$ and the second one is due to the moving pedestrians $\mathbf{F}_{r}^{\text {ped }}$. In case of the existence of other moving obstacles as vehicles, bikes, etc. another repulsive force should have to be incorporated. The final resulting force is:

$\mathbf{F}_{r}=\alpha \mathbf{f}_{r, d}^{\text {goal }}\left(D_{n}{ }^{\text {goal }}\right)+\beta \mathbf{f}_{r, p}^{\text {goal }}\left(D_{n}{ }^{f}\right)+\left(\gamma \mathbf{F}_{r}^{\text {ped }}+\delta \mathbf{F}_{r}^{\text {obs }}\right)$

Where $\alpha, \beta, \gamma$ and $\delta$ are the corresponding weights of the forces. These weights were obtained through two steps: a first approximation of the weights were computed by means of the MCMC-MH method using data of thousands of the simulations of the accompaniment task, and second, these weights were refined by means of an Interactive Learning [56] using data of the real life experiments. For more information about this procedure the reader is referred to [53].
The computation of the $\mathbf{f}_{r, d}^{\text {goal }}\left(D_{n}{ }^{\text {goal }}\right)$ is analog to the attractive force to the destination goal of Eq. 2, but using the actual robot velocity. The repulsive forces from pedestrians and obstacles, $\mathbf{F}_{r}^{\text {ped }}$ and $\mathbf{F}_{r}{ }^{o b s}$ are computed in a similar way as Eq. 3 and 7, but using the robot center position. However, we have to take into account that $\mathbf{F}_{r}^{\text {ped }}$ has to be reformulated because it is now composed by two forces,

$\mathbf{F}_{r}^{\text {ped }}=\sum_{j \in P} \mathbf{f}_{r, j}^{i n t}+\mathbf{f}_{r, c}^{i n t}$

where $\mathbf{f}_{r, j}^{\text {int }}$ are the interaction forces among the robot and other people and $\mathbf{f}_{r, c}$ int is the interaction force between the robot and the accompanied person (the computation of this force is explained in Section 5).

Finally, the new attractive accompaniment force, $\mathbf{f}_{r, p}^{\text {goal }}\left(D_{n}{ }^{f}\right)$ to maintain a formation respect to the companion person is computed assuming that the robot $r$ tries to adapt its velocity within a relaxation time $k^{-1}$, but taking into account the distance and angle to maintain a formation to accompany the person. $D_{n}{ }^{f}$ is the position of the robot respect to the companion person in the next step of the path. The formulation of this force is as follows:

$\mathbf{f}_{r, p}^{\text {goal }}\left(D_{n}{ }^{f}\right)=k\left(\mathbf{v}_{r}^{0}\left(D_{n}{ }^{f}\right)-\mathbf{v}_{r}\right)$

$\mathbf{v}_{r}^{0}\left(D_{n}{ }^{f}\right)$ is the desired velocity vector to reach the configuration position respect to the person at the next step $D_{n}{ }^{f}$, and $\mathbf{v}_{r}$ is the current velocity of the robot. This is the force that keeps the robot in the computed geometrical configuration (robot-human) to allow the joint navigation. It has to be mentioned that the computed geometrical configuration was described in the previous Sub-section 3.2.

The attractive force of the formation $\mathbf{f}_{r, p}^{\text {goal }}\left(D_{n}{ }^{f}\right)$ is applied at each propagation of the position of the companion person along the best path, where $D_{n}{ }^{f}$ is the position of the robot respect to the companion person in the next step. Then, $D_{n}{ }^{f}(t+1)=P^{r}(t+1)$ is the next position propagated of the robot taken into account the constraints from distance and angle (see Fig. 2). The computation of the next position of the robot, $P^{r}(t+1)=\left(\hat{x}^{r}(t+1), \hat{y}^{r}(t+1)\right)$, depends on the next position of the person which is $P^{p}(t+1)=$ $\left(\hat{x}^{p}(t+1), \hat{y}^{p}(t+1)\right)$. The values $\left(\hat{x}^{r}(t+1), \hat{y}^{r}(t+1)\right)$ are computed as follows,

$$
\begin{aligned}
& \hat{x}^{r}(t+1)=\hat{x}^{p}(t+1)+2 R_{i} \cos \left(\theta_{p}-\operatorname{sgn}\left(\theta_{p}-\theta_{c}\right) \theta\right) \\
& \hat{y}^{r}(t+1)=\hat{y}^{p}(t+1)+2 R_{i} \sin \left(\theta_{p}-\operatorname{sgn}\left(\theta_{p}-\theta_{c}\right) \theta\right)(20)
\end{aligned}
$$

Where $2 R_{i}$ is the distance between the robot and the person. $\theta_{p}$ is the person orientation to the destination 
and it is obtained in Eq. 21. $\theta_{c}$ is the actual companion angle between the person's and robot positions. Finally, $\theta$ is the ideal companion angle between the robot and the person calculated in Eq. 12. $R_{i}, \theta_{p}, \theta_{c}$ and $\theta$ are computed in the discrete time $t$. Finally $\theta_{p}$ is computed as follows,

$\theta_{p}=\operatorname{atan}\left(\frac{y^{d}-y^{p}}{x^{d}-x^{p}}\right)$

$\left(x^{p}, y^{p}\right)$ is the detected person position and $\left(x^{d}, y^{d}\right)$ is the position of the final destination for this person, which is inferred amount all the possible final destinations of the environment using the BHMIP method [52], at time $t$. In case the final destination is unknown, the robot predicts the person's path to accompany her/him using a window of time considering its previous orientation.

\section{Robot Approaching People}

Once it has been selected the person of the group that the robot wants to approach, the robot has to calculate the best final position to meet the target person and its correspondent best path to reach this dynamic goal. The dynamic goal takes into account the propagation of the approached person including dynamic and static obstacles. Then, the robot calculates all possible paths and selects the best path to arrive until the dynamic goal, as we explained in the previous section. Also, all the paths take into account the static and dynamic obstacles of the environment. When the group, formed by the robot and the accompanied person, and the target person reach the same goal, the robot needs to rearrange its position respect both persons to be part of the interaction. Part of the approaching task was published on [57].

This section describes two steps: the approaching path, where the robot computes the optimal dynamic destination $D_{n}^{\text {dgoal }}$ (the meeting point) and uses the AKP local planner to compute the best path until this destination, and the proactive configuration to be able of interact with both people.

\subsection{Stage 1: Computation of the Optimal Dynamic Goal}

This subsection describes how the dynamic destination of the group is computed, $D_{n}^{\text {dgoal }}=\left(x^{\text {dgoal }}, y^{\text {dgoal }}\right)$. The dynamic destination, $D_{n}^{d g o a l}$, substitutes the static destination $D_{n}^{\text {goal }}$ of the companion task in Eqs. 1 and 17. Then, we use the companion method of the previous section to calculate all the paths for the group to reach this dynamic destination. The dynamic destination is optimally computed using the gradient descent method, as shown in Alg. 1. This goal optimizes the time to execute the task, and in combination with the AKPcompanion, it also optimizes the traveled distance, the effort of accomplishing the companion task and the effort to avoid obstacles and pedestrians.

Let us describe how Alg. 1 and Alg. 2 work. The Alg. 1 starts at time $t_{n}=0$ and evaluates Eqs. 22 and 23. Then, the iterative part increments $t_{n}$ using the value of the gradient, Eq. 23, and all people's positions and the group's positions are propagated until $t_{n}$ using Alg. 2. $d\left(t_{n}\right)$ and $\frac{\partial d}{\partial t}\left(t_{n}\right)$ are also computed. On the one hand, the propagation of the target person is used as dynamic destination, and, on the other hand, the propagation of the pedestrians in the environment are used to calculate the repulsive forces which affect the trajectory of the group. The outcomes of the algorithm are the minimum encounter time, $t_{n}$, and the optimal dynamic goal, $D_{n}^{d g o a l} \cdot d\left(t_{n}\right)$ is computed as follows:

$d=\sqrt{\left(\hat{x}^{g}-\hat{x}^{t p}\right)^{2}+\left(\hat{y}^{g}-\hat{y}^{t p}\right)^{2}}$

$d\left(t_{n}\right)$ is the euclidean distance between the group and the person to be approached. In Eq. 22 we do not include $t_{n}$ to reduce the formula complexity. $\left(x^{g}, y^{g}\right)$ and $\left(x^{t p}, y^{t p}\right)$ are the positions of the group (personrobot) and the target person, respectively. $\left(\hat{x}^{g}, \hat{y}^{g}\right)$ and $\left(\hat{x}^{t p}, \hat{y}^{t p}\right)$ are the predicted positions for both, group and target person.

$\frac{\partial d}{\partial t}\left(t_{n}\right)$ is the gradient of the distance and is computed as follows,

$$
\begin{gathered}
\frac{\partial d}{\partial t}=\frac{\left(\hat{x}^{g}-\hat{x}^{t p}\right)\left[v_{x}^{g}+a_{x}^{g} t-v_{x}^{t p}-a_{x}^{t p} t\right]}{\sqrt{\left(\hat{x}^{g}-\hat{x}^{t p}\right)^{2}+\left(\hat{y}^{g}-\hat{y}^{t p}\right)^{2}}}+ \\
\frac{\left(\hat{y}^{g}-\hat{y}^{t p}\right)\left[v_{y}^{g}+a_{y}^{g} t-v_{y}^{t p}-a_{y}^{t p} t\right]}{\sqrt{\left(\hat{x}^{g}-\hat{x}^{t p}\right)^{2}+\left(\hat{y}^{g}-\hat{y}^{t p}\right)^{2}}}
\end{gathered}
$$

Where $\left(v_{x}^{e}, v_{y}^{e}, a_{x}^{e}, a_{y}^{e}\right)$ are velocities and accelerations of each entity, $e=\{g, p, r\} . p$ is any person of the environment and $t p$ corresponds to the target person, $g$ is the group, and $r$ is the robot.

In Alg. 2, all predictions until $t_{n}$ are computed using small time intervals. These small increments of time allow the use of constant values of velocity and acceleration. This simplifies the minimization process.

All accelerations are obtained from the interaction with static and dynamic obstacles using the extended social force model (ESFM). The components of this force $\left(F_{x_{t_{i}}}^{e}, F_{y_{t_{i}}}^{e}\right)$ are obtained in Eqs. 1 for the group 

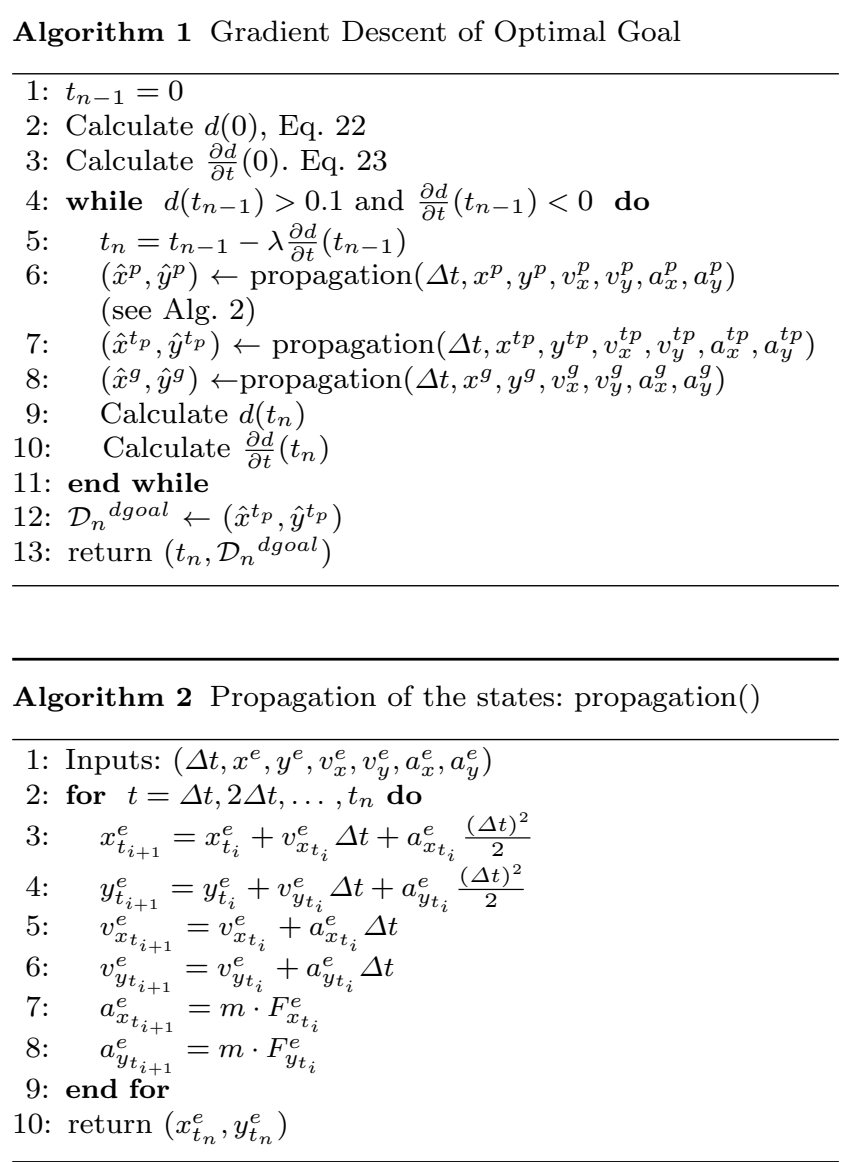

propagation and for people propagation we can use the same formula changing the group $g$ by the correspondent person $p$. As we consider that all the entities have a mass $m=1$, the resulting acceleration is equal to the resulting force.

4.2 Stage 2: Proactive configuration to interact with both people

Once the group and the target person are nearby, the group should face the target person. It is expected that the group and the target person will form a triangle formation. Some previous works use these formation that seems to be natural for humans [58]. To achieve this formation, first, the robot uses the cosine theorem, Eq. 24, to calculate the expected angles of the triangle formation: $\psi_{r}$ is the angle of the vertex that corresponds to the position of the robot, $\psi_{t}$ is the angle of the target person position, and $\psi_{c}$ is the angle of the companion person position.

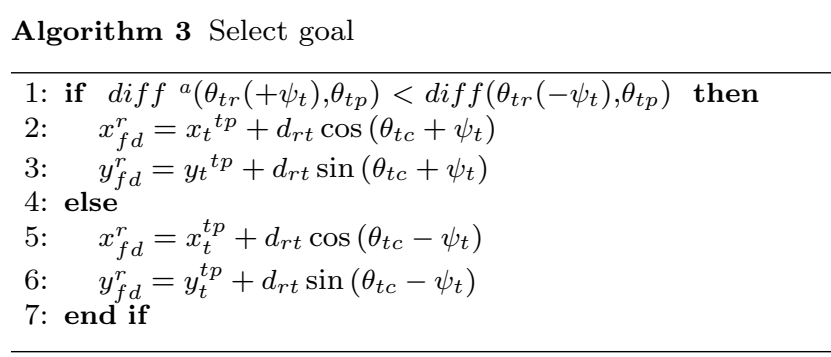

${ }^{a} \operatorname{diff}()$ is a function that calculates the difference between two orientations

Where $d_{t c}$ is the distance between the target person and the companion person, $d_{r c}$ is the distance between the robot and the accompanied person, and $d_{r t}$ is the distance between the robot and the target person. Then, we only fix the distance between the two people and the robot to $1.5 \mathrm{~m}$. We calculate the angle and distance between the two people from its detection's, these values can be any one. Also, the triangle formation gives to us the angles between the two people and the robot (see Fig. 3). Moreover, as we explained previously, we expect to have an ideal distance of 1.5 $\mathrm{m}$ between the robot and the two people, but this can variate depending of the confidence between them and the size of the robot.

Finally, the robot's position is determined using the Alg. 3, which allows the robot to position it self to be able to interact with both people, as it can be seen in Fig. 3 .

where, $\left(x_{f d}^{r}, y_{f d}^{r}\right)$ is the robot's final destination to interact with both people, $\left(x_{t}^{t p}, y_{t}^{t p}\right)$ is the current target person position, $\theta_{t p}$ is the orientation of the target person, $\theta_{t c}$ is the orientation between the target person and the person who accompanies the robot, and $\theta_{t r}$ is the orientation between target person and robot.

\section{ESFM parameter learning for the companion task}

In previous works $[59,60]$ two kind of interaction forces were considered: person with other pedestrians and persons with obstacles. In [53] we added an additional force: person with robots. In this work we have added a new fourth force: robot with accompanied person. This last force shown in Eq. 18 is computed as, 


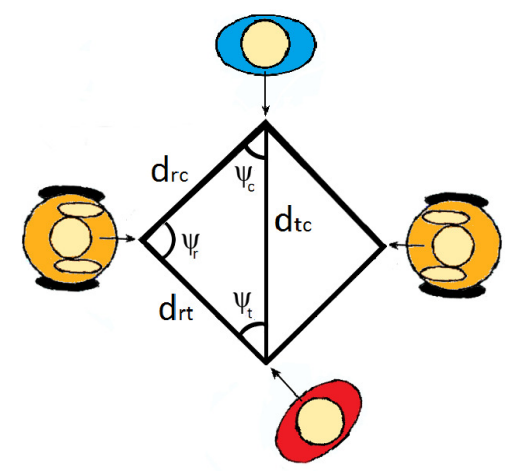

Fig. 3 Triangle positioning of group and target person: Angles and distances that are used in the cosine theorem, and the two possible robot positions to be selected.

$\mathbf{f}_{r, c}^{i n t}=A_{r c} e^{\left(d_{r c}-d_{r, c}\right) / B_{r c}} w\left(\varphi_{r, c}, \lambda_{r c}\right)$

and the new parameters to be learned are $A_{r c}, B_{r c}$, $\lambda_{r c}$ and $d_{r c}$.

To learn these parameters we have created a data base with real-life experiments of two persons doing an adaptive accompaniment task. This means that we have situations where people walk side-by-side and other ones where people have to avoid obstacles while walking together and the group breaks this ideal formation. Our method needs both situations to learn these parameters of the interaction between the members of the group, since the robot performs these types of accompaniment behaviours. The experiments were done with 46 volunteers, which range of ages were among 20 and 75 years old. The data-base includes more than 400 trajectories of two people walking using an adaptive side-by-side formation in an outdoor environment. The setting to obtain the data-base is shown in Fig. 4. The reader can find the database in this link: http://www.iri.upc. edu/people/erepiso/JournalSOR02019_databasePeopleWalking.html

The parameters were learned using a genetic optimization algorithm [61] which minimizes the error between the actual persons companion position trajectory, $x^{p}$, and the expected position for the person companion, $\hat{x}^{p}$, according to Eq. 1. This minimization function is,

$$
\begin{array}{r}
\left\{A_{p p}, B_{p p}, \lambda_{p p}, d_{p p}\right\}= \\
\arg \min _{\left\{A_{p p}, B_{p p}, \lambda_{p p}, d_{p p}\right\}}\left\{\sum_{N} \sum_{\text {time }}\left\|x^{p}(t)-\hat{x}^{p}(t)\right\|\right\}
\end{array}
$$

The resulting parameters of the repulsive force between person-person for the companion task were: $A_{p p}=$

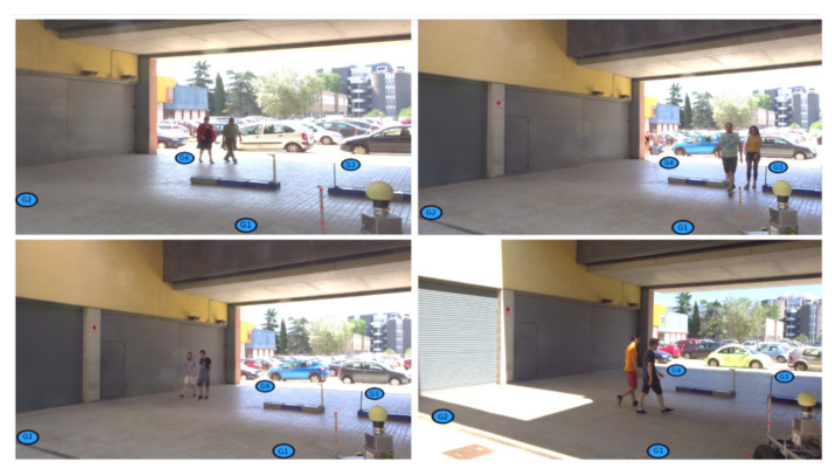

Fig. 4 Setup to obtain the data-base: Four different groups of people walking from one destination to another. The blue ellipses are the destinations of the environment, and the obstacles are a simulated door.

$0.2292, B_{p p}=0.2339, \lambda_{p p}=0.5403$ and $d_{p p}=0.2900$ with a standard deviations of $s t d_{A_{p p}}=0.0065$, std $d_{B_{p p}}=$ $0.0171, s t d_{\lambda_{p p}}=0.0185$ and $s t d_{d_{p p}}=0.0074$. These parameters were used for computation of Eq. 25, although we slightly modified the parameter $A_{p p}$ due that the repulsive force between robot and the accompanied person is higher than between two persons to allow the robot to do not enter in the personal space of the person to be accompanied.

\section{Performance Metrics}

In this section, the performance metrics used to evaluate the robot behaviour are described. All of the metrics are based on previous studies on humans [62] and the proxemic rules, proposed by Hall. [41],

- Intimate distance: Distances between people inside the interval $[0-45 \mathrm{~cm}]$.

- Personal distance: Distances between people inside the interval [ $45 \mathrm{~cm}-1.22 \mathrm{~m}]$.

- Social distance: Distances between people inside the interval $[1.22 \mathrm{~m}-3 \mathrm{~m}]$.

- Public distance: Distances between people are 3 meters away $(>3 \mathrm{~m})$.

Usually, the companion and approaching behaviours between people are inside the area of personal distances. However, to take into account the size of our robot and interactions with people unfamiliar with robots, we expect that the distances between robot and people will be inside the area of social distances. Note that all of our distances have been calculated from the center of the robot to the center of the person and that these distances include the radius of both and a free space between them. 
6.1 Performance metrics for the companion task

The companion task has been evaluated using three types of performance metrics. The first metric, area performance metric, is composed by three areas shown in Fig. 5-left and it is used to evaluate if the robot is in the appropriate companion area. The second and third performance metrics serve to differentiate if the companion task is failing on the distance between both or on the ideal angle of companion, see Fig. 5-right.

The first metric takes into account the spatial 2D relation between the robot and the person and it is defined by three areas. (i) Human's personal space $\mathcal{C}$, is the area where the robot can not be in order to avoid invading human's personal space and it takes into account the minimum person radius space and some free space, correspondent to $R_{i}$ (see section 3.2). (ii) Social distance area $\mathcal{A}$, is the area where the robot should be to be socially accepted. And (iii) Human's companion area $\mathcal{B}$, is the area where the robot has to be to accompany the person. In our case, the last area is a dynamic area inside the social distance that is computed at every accompaniment robot position (see section 3.2 ). To obtain the limit of this area we consider the double of the robot radius, because the robot has some margin with respect to the ideal position to allow not do $\mathrm{S}$ behaviour. The computation of these areas is shown below.

$$
\begin{aligned}
& \mathcal{A}=\left\{x \in \mathbb{R}^{2} \backslash(\mathcal{B} \cup \mathcal{C}) \mid 0.75<d\left(x, p_{c}\right)<3\right\} \\
& \mathcal{B}=\left\{x \in \mathbb{R}^{2} \backslash \mathcal{C} \mid d\left(x, r_{c}\right)<1\right\} \\
& \mathcal{G}=\left\{x \in \mathbb{R}^{2} \mid d\left(x, p_{i}\right)<0.75\right\}
\end{aligned}
$$

where $p_{c}$ is the actual position of the person who accompanies the robot, $p_{i}$ is the position of any pedestrian, and $r_{c}=P^{r}(t)=\left(x^{r}(t), y^{r}(t)\right)$ is the ideal position of the robot. This position is computed using Eq. 20. In the area $\mathcal{A}$, we consider that if the distance is greater than 3 meters, a person does not feel that the robot is accompanying him.

Moreover, the robot has a radius of $0.5 \mathrm{~m}$ and can be represented as a circle of $1 \mathrm{~m}$ of diameter, with center on the robot's position $r, \mathcal{R}=\left\{x \in \mathbb{R}^{2} \mid d(x, r)<0.5\right\}$, whose area is $|\mathcal{R}|=\frac{\pi}{4}$.

The area metric for the companion task can be computed for each human's position $p_{c}$ and robot's position $r$, and is formulated as follows:

$\mathrm{P}\left(r, p_{c}\right)=\frac{1}{|\mathcal{R}|} \int_{\mathcal{B} \cap \mathcal{R}} d x+\frac{1}{2|\mathcal{R}|} \int_{\mathcal{A} \cap \mathcal{R}} d x \in[0,1]$
The metric has the maximum performance when the robot is in the area described by $\mathcal{B}$, since it is the best position to accompany the human in each instant of time. Additionally, if the robot is in the area $\mathcal{A}$, but not in area $\mathcal{B}$, is a partial success, since the robot is inside the social distance accompanying the human, but not in the best accompaniment position. Finally, if the robot is further than three meters from the human's position, then we consider that there is no companionship interaction between robot and person, and therefore its performance is zero. Also, if the robot invade any human's personal space is penalized with zero performance.

The functions of the distance and angle performance metrics are displayed in Fig. 5-right. We consider that the robot achieves a good distance performance if it keeps its central position inside the interval of distances $[1.25-2] \mathrm{m}$, respect to the position of the accompanied person. This margin is centered in the ideal value of $1.5 \mathrm{~m}$. This margin decreases the tolerance of 0.25 $\mathrm{m}$ from the ideal value; and increases $0.5 \mathrm{~m}$ more for larger distances, because some times the person moves away the robot and needs to correct its position to approach it. The equation of the distance performance metric is shown in Eq. 29, where $P_{2 R_{i}}$ means distance performance. In terms of angle, the best angle performance is, at most, a difference of $10^{\circ}$ from the ideal angle to accompany the person and then the angle performance value is 1 . Then, if the difference of the angle increases, we penalized each increment of $10^{\circ}$ with -0.1 in the angle performance, to obtain a 0 value, when the robot has an error of $90^{\circ}$ with respect to the ideal value. The equation of the angle performance metric is shown in Eq. 30, where $P_{\theta_{\text {diff }}}$ means angle performance and $\theta_{\text {diff }}=\left(\theta(t)-\theta_{r}(t)\right)$, and $\theta(t)$ means the ideal angle of companion and $\theta_{r}(t)$ is the real companion angle between the robot and the person.

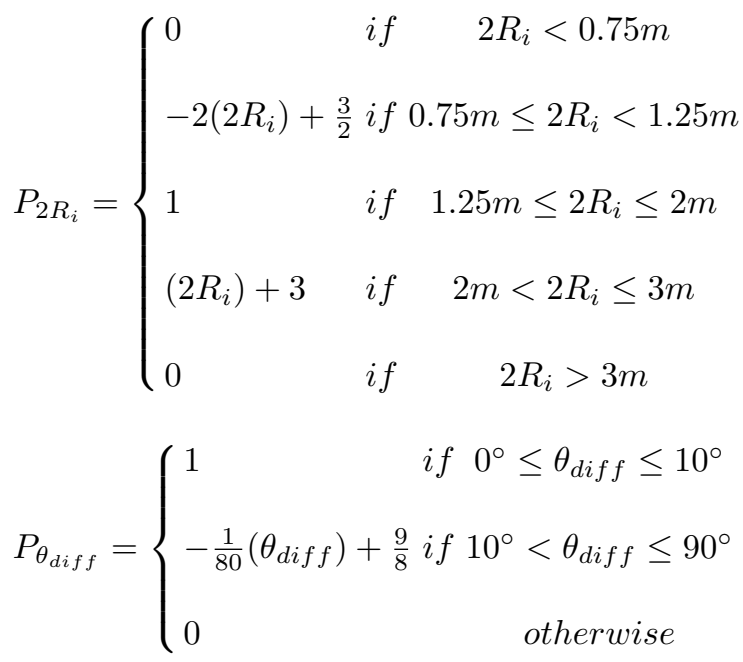



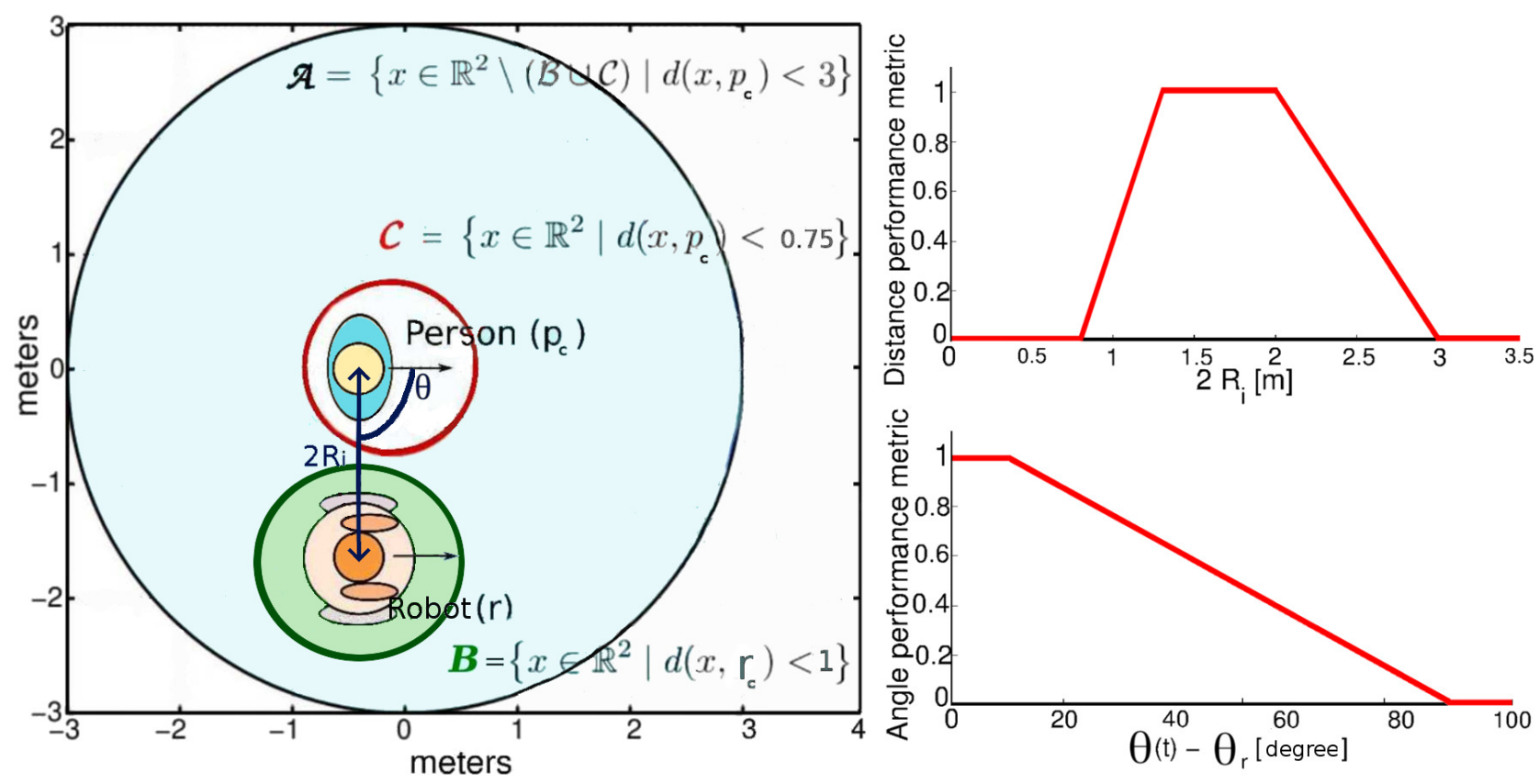

Fig. 5 Metrics of area, distance and angle performance for the companion task. Left: Diagram of the areas for the evaluation of the area performance metric. The performance has value of 0.5 , when the distance between the robot and the accompanied person is in the interval of [0.75-3] meters. If the robot is inside the area of 1 meter around the ideal position to accompany the person, the performance increase to 1 . This ideal position is obtained with the distance between them $2 R_{i}$, fixed at the ideal value of $1.5 \mathrm{~m}$, and the ideal angle $\theta$, obtained with Eq. 12. Right: Performance metrics for distance and angle. Top: plot of the metric of performance in distance and Bottom: plot of the metric for the angle performance. The value of performance equal to 1 is considered the best value.

\subsection{Performance metrics for the approaching task}

Our goal is to evaluate the approaching behaviour between the group and the person. We expect that the distance between the group and the target person decreases during this part of the whole task. Also, the distance of the computed global path of the robot has to decrease. Then, the evaluation of the approaching task was done by imposing two conditions: One that shows the distance between the group and the target person, and other that shows the distance of the global path until the dynamic destination. An example of these graphs can be seen in Fig. 9 .

\subsection{Metrics of Performance for the Final Positioning}

The final position of the robot respect to both people has been evaluated using three type of performance metrics. One related with proxemics, based on several areas of performance, see Fig. 6-left. And two metrics that serves to differentiate if the final positioning task of the robot is failing in the distance between the target person or in the ideal angle between the target person and the robot, see Fig. 6-right. Remember, that the dis- tance and angle between the robot and the companion person were evaluated on the performance for the companion task.

For the first performance, two of the previous areas are used, $\mathcal{A}$ and $\mathcal{C}$. Furthermore, one new area must be defined: $\mathcal{E}$, which is the best robot's position of the final positioning task. The area $\mathcal{E}$ is centered in a position where the robot performed a triangle formation with both people, to be an active part of the final group. Also, we have to duplicate area $\mathcal{A}$ and $\mathcal{C}$ to the center of each person position: accompanied person, areas $\mathcal{A}_{1}$ and $\mathcal{C}_{1}$, and target person, areas $\mathcal{A}_{2}$ and $\mathcal{C}_{2}$. The computation of $\mathcal{E}$ is,

$\mathcal{E}=\left\{x \in \mathbb{R}^{2} \backslash \mathcal{C} \mid d\left(x, r_{a}\right)<1\right\}$

Where $r_{a}=\left(x_{d}^{r}, y_{d}^{r}\right)$ is the desired final position of the robot to be in a triangle formation with both people, the target and the companion person. This position is obtained using the cosine theorem of the Alg. 3. The limits of the areas are analog to the case of the performance metrics for the companion task.

The area performance for the final positioning task is analog to Eq. 28, but now, it has the maximum performance value inside the area described by $\mathcal{E}$, since 

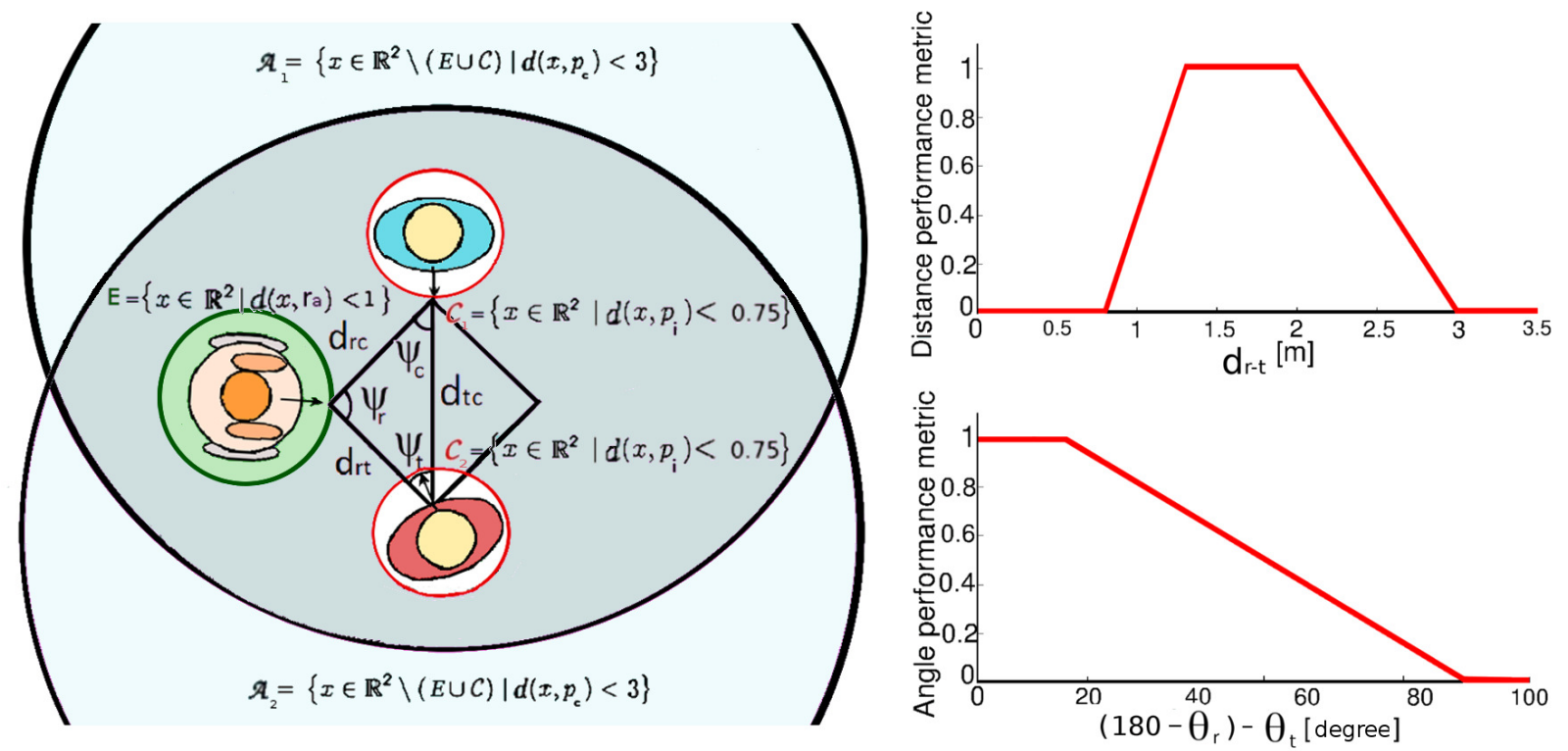

Fig. 6 Metrics of area, distance and angle performance for the final positioning task. Left: Metric of area performance: The performance has value of 0.5 , when the distances between both people and the robot are in the interval of [0.75-3] meters. If the robot is inside its position of the triangle, calculated with the cosine theorem of the Alg. 3, the performance increases to 1, but always outside of the personal space of all of the people. Right: Metrics of performance in distance and angle. Top: plot of the metric of performance in distance. Bottom: plot of the metric of performance in angle. The value of performance equal to 1 is considered the best value.

it is the best position to face the target person while the robot is positioned to have equal distance to both people. Additionally, when the robot is inside the area $\mathcal{A}_{1} \cap \mathcal{A}_{2}$, it is a partial success, since the robot is inside the social distances of both people, but not in the $\mathcal{E}$ area. Finally, if the robot is further than three meters of the final positioning, we consider that the robot is not doing the task correctly, because people can feel that the robot is not part of the group, and therefore its performance is zero. Also, if the robot invades any human's personal space is penalized with zero performance.

The performance of distance and angle of the final positioning task are similar to the performances of the companion task Fig. 5 and Eq. 29 and 30. We consider that the robot makes a good performance if it keeps its central position inside the interval of distances [1.25-2] $\mathrm{m}$, with respect to the position of the target person. Furthermore, for the orientation angle, we have considered a good performance when the orientation of the group differed as much as $15^{\circ}$ from the orientation to face the center of the group. Finally, we obtained that the robot reduces the difference of orientations between the two people if it is oriented to the center of the group, and both people feel that the robot is part of the final group.

\section{Synthetic Experiments}

A complex dynamic simulation environment was developed to reproduce real-life situations. The simulation environment consists of a set of static obstacles and also includes the motion of several pedestrians. These simulated human's used the ESFM to obtain a more realistic navigation behaviour and a random velocity inside the interval $[0-1] \mathrm{m} / \mathrm{s}$. Furthermore, the simulated environment includes the Tibi robot model which uses our companion, the approaching and final positioning methods. The simulated robot is non-holonomic and has a maximun velocity of $1 \mathrm{~m} / \mathrm{s}$, and it adapts to the velocity of the accompanied person. In addition, the accompanied person uses the same navigation and dynamic goal computation as the robot, to obtain a quite similar human behaviour in the accompaniment and approaching tasks. This behaviour includes the avoidance of static obstacles and dynamic people in a more realistic way.

More than 4,700 simulations were performed to test and validate our model. The method was tested in a dynamic uncontrolled situations, where we had static and dynamic obstacles at the same time. Also, we included a large number of people that walks randomly towards any destination. In addition, the orientations and speeds of the approximation between the group and 


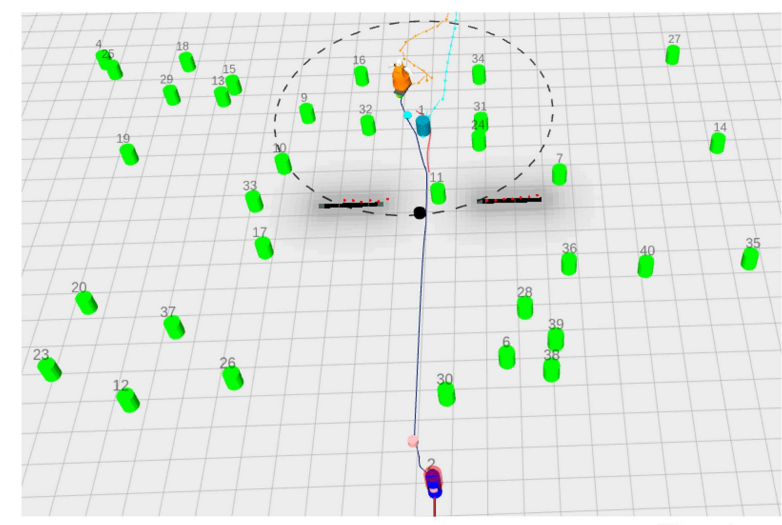

Fig. 7 Synthetic experiments: Simulation environment to test and evaluate the companion, approaching and final positioning tasks. The black rectangles are the static obstacles and the green cylinders are several people. Furthermore, the environment has the Tibi's model, the accompanied person in blue, and the person that they want to approach in red.

the target person were changed, to represent as many real-life cases as possible. With these simulations, we covered a great range of real-live situations, for example, we included different person crosses, static and dynamic passage ways and multiple interactions with several people and static obstacles at the same time. These situations manifest the good performance of the rearrangement of the group while navigates in dynamic environments, moreover the computation of the dynamic goal takes into consideration the avoidance of dynamic and static obstacles and test the robustness of our algorithm in complex situations. Fig. 7 presents the simulation environment.

The reader can see the results of the companion and final positioning task in Table. 1 and in Fig. 8-left and Fig. 8-right, respectively. The results are expressed in a scale between 0 and 1 , where 1 is the best value. The value between brackets is the standard error of each mean value. Also, we have to take into account that the area performance is more restrictive than the distance and angle performances, because we expect that all the area of the robot will be inside the correct area to accompany the person.

Regarding the approaching task for all the simulations, we obtained several graphs similar to Fig. 9. Notice how the distance between the group and the target person decreased in each iteration, Fig. 9-left, or how the distance of global path until the dynamic destination also decreased, Fig. 9-right. These results exhibit a good performance of the approaching task.

During the simulations, we saw several behaviours that seems an intelligent robot. As for example: in most of the cases the robot surrounded the person to avoid other people and went to the other side, where it had free space. These behaviours were not explicitly implemented but emerge naturally, where the robot tries to find the position next to the accompanied person that has more free space. Also, we found several temporal local minima in simulations, when we had high density of random people, that blocked the path of the pair. Besides, in these situations the simulation environment and our algorithm showed a very realistic behaviour, where the group stopped momentarily and waited until the other pedestrians continued their way to their destinations and left some free space, and then the group continued the walk until met the target person. This behaviour was represented in the results obtained for the approaching task in Fig. 9-right. In this graph, we had some points, where the distance of the global path until the dynamic destination remain constant for a while and after some time continue decreasing, this also means that the distance between the group and the target person remained standing for a while an after that, the distance between them decreases again.

\section{Real-Life Experiments}

The proposed method was also tested in real-live experiments, with Tibi, the robot of the Institut de Robotica i Informatica Industrial (IRI). The maximum robot speed was set up to $1 \mathrm{~m} / \mathrm{s}$ due to security reasons, but it can adapt to the velocity of the accompanied person. For allowing the robot to adapt to people velocity, we imposed $0.8 \mathrm{~m} / \mathrm{s}$ as the maximum speed of people. We used different dynamic urban environments that will be presented on Sub-sec. 8.1. Also, the robot platform is described in Sub-sec. 8.1. We made three different blocks of experiments: to evaluate the companion task Sub-sec. 8.3, to evaluate the entire task with companion, approaching and final positioning at the same time Sub-sec. 8.4, and an user study of the whole task Sec. 9. In addition, the reader can find several videos of the real experiments in this link: http://www.iri. upc.edu/people/erepiso/JournalSOR02019.html

\subsection{The Robot Platform}

To carry out all the real-life experiments we used the Social robots Tibi and Dabo, Fig. 10-Left. They are the two semi-humanoid social robot's of the IRI, developed in the URUS project [63].

These robots were developed to share dairy tasks with people in urban areas. To do it, they have nice 

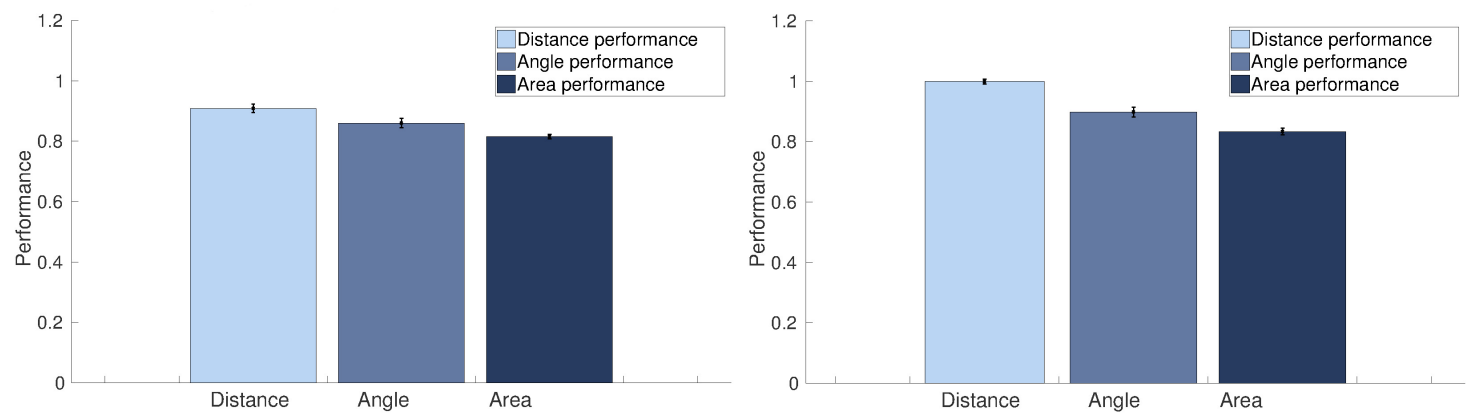

Fig. 8 All performance for the companion and final positioning tasks. Left: Performance's of the companion task. Right: Performance's of the final positioning task. Inside the two graphs, the first column is the distance performance, the second is the angle performance and the third is the area performance. These columns show the average and the standard deviation of performance for all simulations. The performance value equal to 1 is considered the best value.

\begin{tabular}{|l|c|c|}
\hline & Performance of Companion & Performance of Final Positioning \\
Mean of Distance Performance & $0.91( \pm 0.01)$ & $1( \pm 0.0078)$ \\
Mean of Angle Performance & $0.86( \pm 0.015)$ & $0.9( \pm 0.016)$ \\
Mean of Area Performance & $0.82( \pm 0.0073)$ & $0.84( \pm 0.011)$ \\
\hline
\end{tabular}

Table 1 Performance results of the simulation experiments for the tasks: companion and final positioning. The performance value equal to 1 is considered the best value and the values between brackets are the standard errors of each mean value.
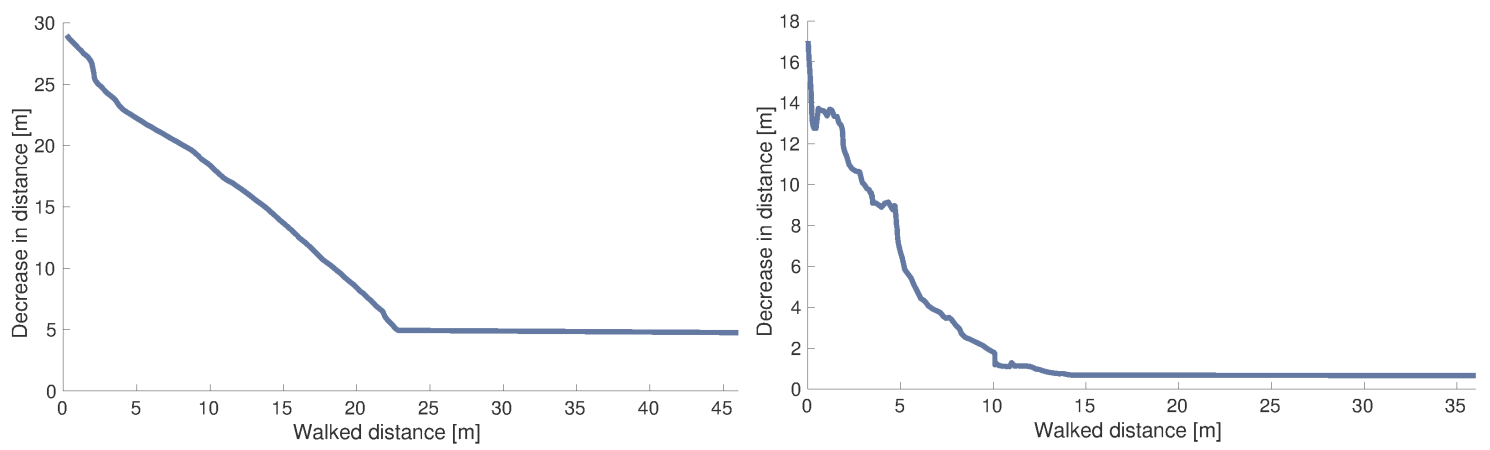

Fig. 9 Performances for the approaching task. Left: Graph of the difference in distances between group and target person. Right: Graph of the distance of the global path until the dynamic destination. In both graphs we see the reduction of the distances in each iteration. In the graph of the global path we see how the distance of the path remained standing for a while an after that decreases again. These situations are done because other people block the path of the group in several points during a small periods of time.

appearance and multiple sensors to be able to interact with people.

They have a two-wheeled Segway RMP200 platform to move around the environment. They have several sensors to perceive its surroundings, as two Hokuyo UTM-30LX 2D laser range sensors (front and backward), to build maps, to be localized in the environment, to detect obstacles and to track people.

Also, the robots have different visual sensors as the lady bug and the bumbelbee stereo cameras, to detect objects and track people of the environment. Further- more, the robots have different social interaction elements as a tactile screen to receive commands through a menu or visualize results, a speaker to get spoken interaction, two mobile arms to perform non verbal communication and LED's in their faces to represent face expressions. All the programs that control the robot behaviours were executed inside two onboard computers (Intel Core 2 Quad CPU @ 2.66 and $3.00 \mathrm{GHz}$ ) and its power is supplied by two sets of batteries, one for the Segway platform and one for the computers and sensors. We used a laptop to monitor the robot behaviour 


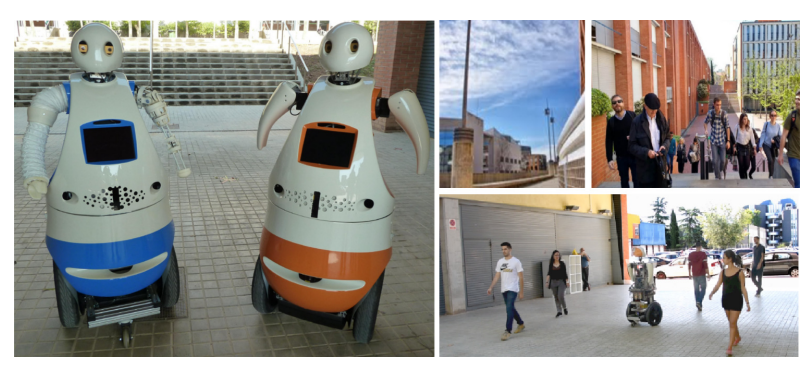

Fig. 10 Robotic platforms and environments. Left: Tibi and Dabo the two humanoid robots of the Institut de Robotica i Informtica Industrial (IRI). Right: The upper images correspond to the Barcelona Robot Lab (BRL) situated at UPC North campus, and lower image correspond to the Facultad of Matemtiques i Estadstica Lab (FME) at the UPC South Campus.

in all of the experiments. We used Ubuntu-linux Operating System with several algorithms coded on $\mathrm{C}++$ and use ROS as a middleware. The companion system run at $5 \mathrm{~Hz}$ that allowed the robot to react quickly to different situations and changes in the environment.

These robots could do several interactions or collaborations with humans, such as: find pedestrians or things, recognize humans, navigate alone or together, follow, guide or accompany people.

\subsection{The Experimental Environments}

The real experiments were carried out in two outdoor urban areas: Barcelona Robotics Laboratori

(BRL, Fig. 10-Upper Right) and the Facultad of Matematiques i Estadistica Lab (FME, Fig. 10-Lower Right). The BRL is situated at the UPC North Campus in Barcelona. This environment is a University campus area of over $10,000 \mathrm{~m}^{2}$ that includes several buildings, squares, staircases, ramps and narrow passages, as well as multiple static and dynamic obstacles like: flowerpots, waste bins, banks, trees, bicycle stands and people. The FME is situated at the UPC South Campus in Barcelona and is a free area of $15 \times 15$ meters delimited by a stairs, two walls and a street.

\subsection{Companion Task Real-Life Experiments}

The experiments of the companion task were done by Tibi robot in the square yard of the FME. Several volunteers were accompanied to different destinations, while the group had to avoid static and dynamic obstacles. In these experiments, the method was evaluated with the performance metrics for the companion task.

\begin{tabular}{|l|c|}
\hline & \multicolumn{1}{|c|}{ FME } \\
& $\begin{array}{l}\text { Performance of } \\
\text { Companion }\end{array}$ \\
Mean of Distance Performance & $0.91( \pm 0.0094)$ \\
Mean of Angle Performance & $0.73( \pm 0.0018)$ \\
Mean of Area Performance & $0.63( \pm 0.012)$ \\
\hline
\end{tabular}

Table 2 Performance results of the real-life experiments carried out in the FME to test the companion task. The performance value equal to 1 is considered the best value and the values between brackets are the standard errors of each mean value.

We developed five sets of experiments that include a huge range of real situations. First, in a free area without any kind of obstacles. Second, some static obstacles were included to increase the difficulty. If the robot was able to pass throw a door with the person it accompanies, also was able to avoid an obstacle in one of the person sides. Third, we included the avoidance of several groups of pedestrians. Four, the robot navigated in an environment with obstacles and pedestrians. Last, a crowded environment with many obstacles was considered in order to increase the difficulty of the experiments.

The results of the companion task are shown in Table. 2. Notice that the results are expressed in a scale between 0 and 1 , where 1 is the best value. The value between brackets is the standard error of each mean value. Also, we have to take into account that the area performance is more restrictive than the distance and angle performances. Finally, we obtained an acceptable performance in spite of the difficulty of the task in the reallife experiments. Images of the experiments are show in Fig. 11-Top.

\subsection{Companion, Approaching and Final Positioning} Tasks Real-Life Experiments

The whole task was tested on real-life experiments in the FME with Tibi. The whole task included: companion, approaching and final positioning of the robot. In these experiments we assumed that the two people knew each other and they wanted to get closer to interact. Also, we expected that the robot knew previously both people and knew which person had to accompany and to which person had to approach. Then, we selected exactly the person to accompany and the person to approach, because the autonomous recognition and differentiation from other people is out of the scope of the work. All people were detected using a laser leg detector and tracked using a multi-hypothesis tracker, published in [64]. 


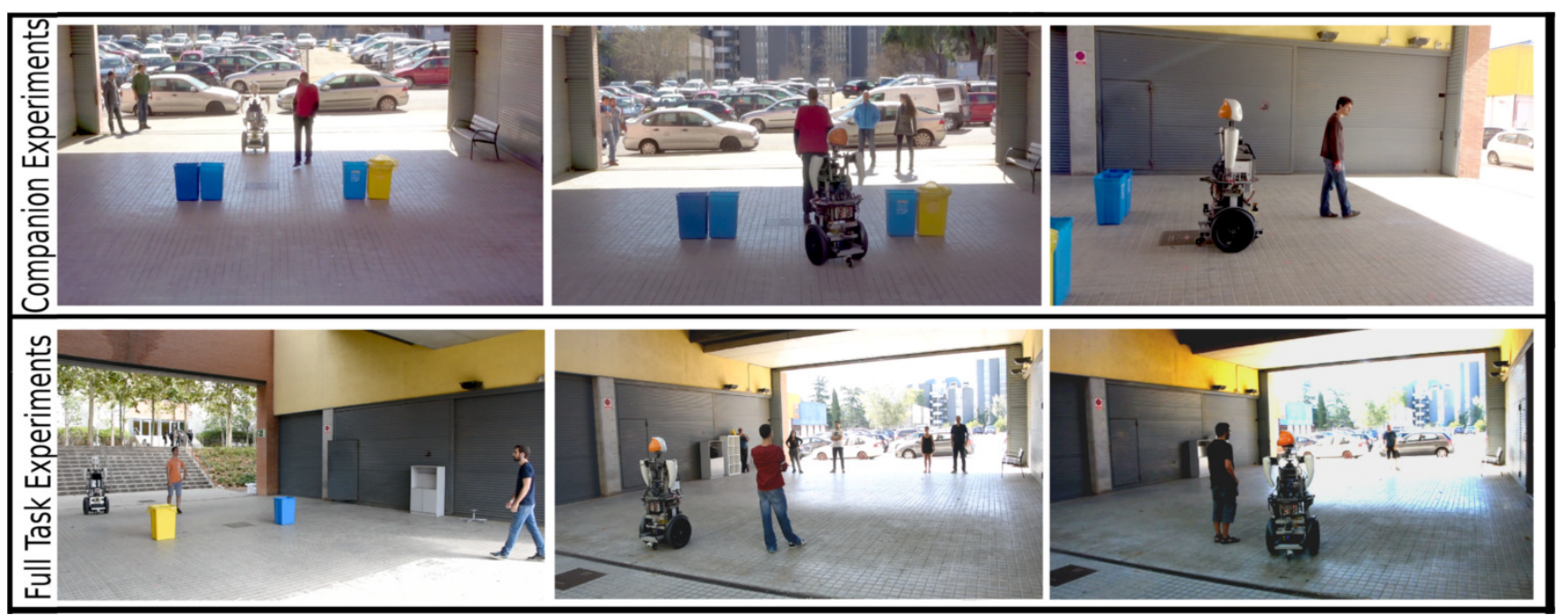

Fig. 11 Real-life experiments. Top: The images show different moments of the real experiments for only the companion task. Bottom: The images show several moments of the real-life experiments for the full task: accompany, approach and final positioning. In both cases the robot has to perform the task while avoids other people and obstacles.

We developed the same five sets of experiments of the companion case, but this time the final destination was dynamic, it corresponded to the movement of the target person that the group wanted to approach. Furthermore, for each one of the five sets, we developed a significant set of different meeting orientations between the group and the target person. After all, we obtained a huge range of possible companion, approaching and final positioning interactions. We can see three representative images of these experiments in Fig. 11-Bottom.

The reader can see the results for the companion and final positioning tasks of the FME in Table. 3. The results are expressed in a scale between 0 and 1 , where 1 is the best value. The value between brackets is the standard error of each mean value. For the results of the approaching task we obtained similar graphs to the ones obtained in the simulations, Fig. 9, where we could see how the distance between the group and the target person decreased during time.

\section{User Study}

To develop the user study, we performed 72 real-life experiments: 36 using our method and 36 controlling the robot by teleoperation. The method and the teleoperation has the same limit of maximum velocity of $1 \mathrm{~m} / \mathrm{s}$. The teleoperator used the following rules: do not disturb the accompanied person or other pedestrians in the environment, perform a side-by-side accompaniment, approach to the target person, try to imitate human side-by-side behavior and approaching be- haviour. These experiments were made in the North Campus with Tibi. The participants were 72 inexpert users. The average and standard deviation of the ages of the participants was $(\mathrm{M}=27.21, \mathrm{SD}=11.72)$, and the range of ages were between [15-76] years old. The $25 \%$ were women and $75 \%$ men, mainly university students and some workers of the university campus, see Fig. 1. The level of knowledge in the robotics field was included in the surveys and was ranged between 1 to $7.65 \%$ of the participants had the lowest level of knowledge in robotics (1,2 and 3), 18\% had the intermediate level of knowledge in robotics (4 and 5) and $17 \%$ had the highest level in knowledge of robotics (6 and 7). Then, we can conclude that the highest amount of the participants were potential users not related with robotics.

In the experiments, Tibi was accompanying one of the participants and both met another person. Different approach directions were performed during the experiments. In addition, the final positioning was improved avoiding unnecessary movements of the robot. Now, the robot does a more human-like behavior, by positioning itself in the free space of the triangle closest to its position. After that, the robot turns towards the center of the group. This behavior assumes that both people will be turned towards the center of the group, as humans normally do. Furthermore, other pedestrians were walking around the campus or watching the experiment, but none interfered directly in the way of the group. In cases where we only had an inexpert person, he or she accompanied Tibi and one of our technicians played the role of person who would meet the group. 


\begin{tabular}{|l|c|c|c|c|}
\hline & \multicolumn{1}{|c|}{$\begin{array}{c}\text { FME } \\
\text { Performance of Companion }\end{array}$} & $\begin{array}{c}\text { FME } \\
\text { Performance of the Final Positioning }\end{array}$ \\
\hline Mean of Distance Performance & $0.87( \pm 0.03)$ & $0.84( \pm 0.024)$ & $0.84( \pm 0.019)$ & $0.87( \pm 0.029)$ \\
Mean of Angle Performance & $0.68( \pm 0.04)$ & $0.7( \pm 0.022)$ & $0.97( \pm 0.0025)$ \\
Mean of Area Performance & $0.73( \pm 0.067)$ & $0.65( \pm 0.039)$ & $0.84( \pm 0.017)$ & $0.83( \pm 0.022)$ \\
\hline
\end{tabular}

Table 3 Performance results of the real-life experiments carried out in the FME and the BRL of the whole task. Here, we show the results of companion and final positioning. The results of the approaching task are similar to Fig. 9. The performance value equal to 1 is considered the best value and the values between brackets are the standard errors of each mean value.

\begin{tabular}{|l|}
\hline Survey's Questions \\
\hline Robot's Intelligence Scale \\
How intelligent did the robot behave? \\
How well did the robot anticipate to your's and \\
other's movements? \\
\hline Robot's Sociability Scale \\
How natural was the robot's behaviour? \\
How interactive was the robot's behaviour? \\
\hline Robot's Comfortableness Scale \\
How comfortable did you feel near the robot? \\
How safe did you feel around the robot? \\
How well the robot conserve your physical space? \\
\hline
\end{tabular}

Table 4 Questionnaire. Survey questions asked of each participant. All questions were asked on a 7-point scale from "Not at all" to "Very much".

One example of the real-life experiments of this section is shown in Fig. 1.

The results of the real-life experiments for the Companion and Final Positioning tasks in the BRL are shown in Table 3. These results demonstrate that the robot is able to approach people while accompanies a pedestrian. A user study was also conducted to determine whether the use of the ESFM enhances the base-line model, this is, an expert moved the robot using teleop, and we should highlight that people perceived a difference between these two approaches.

Participants were asked to complete a variety of surveys. It was randomly selected if whether the robot autonomously accompanied a person and approached another pedestrian or if it was moved using teleop.

Social Scales. Participants were asked a set of questions, as shown in Table 4, following their encounter with the robot in each mode of behaviour. To analyze their responses, we grouped the survey questions into three scales: the first measured the robot intelligence, while the second and third evaluated robot's sociability and comfortableness felt by the volunteers. Both scales surpassed the commonly used 0.72 level of reliability (Cronbach's alpha).

Each scale response was computed by averaging the results of the survey questions comprising the scale. ANOVAs were run on each scale to highlight differences
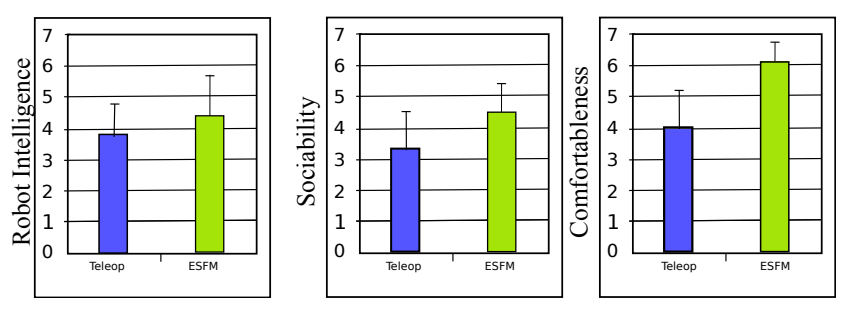

Fig. 12 User study results. Left: Robot's Intelligence. Center: Robot's Sociability. Right: Robot's Comfortableness.

between the two robot behaviours, plotted in Fig. 12. For the robot's intelligence score, plotted in Fig. 12Left, pairwise comparison with Bonferroni demonstrates no statistical difference between the two kind of navigation approaches, $p>0.5$. In terms of robot's sociability and comfortableness the volunteers also perceived a difference between the two navigation's, $p<0.01$ in both cases.

Therefore, after analyzing these three components in navigation terms, we may conclude that if the robot has the ability to socially navigate and respect human conventions using our ESFM, it has the largest acceptance as people perceived the robot to be more comfortable and more sociable.

People perceived our method as better than the teleoperator, because the method kept a comfortable distance for inexperienced people, since the robot tried to keep a free space between him and the person of $0.7 \mathrm{~m}$, (it is an exact distance of $1.5 \mathrm{~m}$ between the center of the robot and person positions, tacking into account that the Tibi's radius is $0.5 \mathrm{~m}$ and a normal person radius is $0.3 \mathrm{~m}$ ) that is inside the interval of personal distance in proxemics, and many people feel uncomfortable when the robot was more close to them. Few people complained about the larger distance of our method, only people more extroverted or who liked the robots considered that Tibi kept too much distance between them. We select that distance based on the radius of the robot, the person's radius and a study in a previous work [7]. To use the findings of this previous work, we take into account that our method performs 
an adaptive side-by-side that allows the robot to position itself at front, at lateral and at back of the person who accompanies depending on the situation. In the triangle of the final positioning, the perception of the people was also better for the method because it was more conservative quipping the exact distance and orientations of the triangle formation that they perceived as more comfortable and natural behaviour.

\section{Discussion}

The findings presented in the previous section reinforce the notion that the robots ability to collaborate with people by approaching and accompanying them is an important skill, and one that is necessary for engendering better collaborations between people and robots. In general, some participants were surprised to find a robot on the campus premises, while others who were familiar with Tibi were happy to see it again. Additionally, most people were very impressed when Tibi was able to respond to their behaviour by turning at appropriate moments or stopping suddenly in response to human stopping movements.

Volunteers seemed to favour our method over the teleoperation method, because they feel more comfortable to the exact distance conserved by the method and this behaviour seems to them that the robot reacts quickly to its movements, creating a better experience of human-robot interaction.

We also had success demonstrating the method's suitability within dynamic urban environments during the accompanying and full task experiments of the FME, wherein Tibi was able to accompany a person while avoiding static and dynamic obstacles and responding spontaneously to unexpected situations. These participants were familiar with Tibi and its behaviour, but could still appreciate the robot's agility in anticipating their movements or reacting to unexpected situations. In terms of the full task performance, Tibi was able to gauge the meeting point in advance and anticipate the human's movements and actions accordingly, ultimately carrying out the planned interaction with two people.

We observed that in larger spaces, Tibi's speed limitations seemed to be excessive, and younger participants had to slow their pace in order to be accompanied by the robot. Also, many volunteers suggested that it would be interesting to incorporate additional social interactions into the accompaniment task, such as talking or making gestures. These remarks will be considered in our future research endeavours.

Also, we found some differences between people of different cultural backgrounds. Then, we suggesting that robots might also need to learn to adapt and modify their behaviour to more suitably interact with the people it encounters in a way that meets their cultural preferences or expectations.

\section{Conclusions and Future Work}

In this work, we present a new framework for how autonomous robots approach and accompany people in urban environments. The method discussed allows the robot to accompany a person and approach to other one, by adapting its own navigation in anticipation of future interactions with other people or contact with static obstacles. The major contributions of this paper are manifold:

Firstly, the model for how a robot accompanies a person is based on the SFM (Social Force Model), where we have introduced new forces to cope with this task. Two new forces have been introduced in the accompaniment task, one attractive to maintain the adaptive side-by-side formation and one repulsive to maintain the security distance between the robot and the human in this task. The new attractive force uses a dynamic virtual goal to keep the adaptive companion position around the accompanied person, that serves to accompany in a side-by-side formation and change this formation when the group needs to avoid dynamic and static obstacles.

With this new model, we have extended the Anticipative Kinodynamic Planner for the side-by-side formation in complex environments, allowing not only to be applied to one robot and one person, but also to many robot and many persons.

Secondly, the model for how a robot approaches another person, and interacts with both, is also based on the SFM (Social Force Model) and in the results of the previous accompaniment method, but now we have introduced a dynamic destination goal and adapted to our necessities the triangle formation for the approaching of the group to the target person.

In these two new results, we have make extensive use of dynamic virtual destinations in the attractive forces, which we already introduced in our previous work. It is important to comment that this dynamic virtual destination open the door and extend the ESFM to new types of interactions among robots and humans.

Finally, we have created a database of peoples trajectories that can be accessed and used by other researchers. Using this database we obtain the interaction parameters for the robot and the companion person. The results of the real life experiments shows that a social robot is able to successfully accomplish the companion, approaching and final positioning tasks. The 
method was extensively and rigorously tested in a reallife environments in Barcelona, with non-trained volunteers. Our user study showed that the method was generally acceptable to laypeople with no expertise in the subject.

In order to continue the work presented in this paper, we plan to develop new techniques to customise the robot's accompaniment task, and adjust its approach in accordance with human preferences. This entails allowing the robot to learn different values for the distance/velocity of different people with diverse personalities or skill sets, during the accompanying task. Using this knowledge, the robot could recognise the kind of person interacting with it, and adjust its behaviour in accordance with his or her preferences. By enabling this level of customisation, we aim to respond to some of the problems we encountered during the user study, wherein different kinds of people favoured different distances or speeds while interacting with the robot.

\section{Acknowledgments}

Work supported by the Spanish Ministry of Science and Innovation under project ColRobTransp (DPI201678957-RAEI/FEDER EU) and by the Spanish State Research Agency through the Maria de Maeztu Seal of Excellence to IRI (MDM-2016-0656). Ely Repiso is also suported by Spanish Ministry of Science and Innovation under a FPI-grant, BES-2014-067713.

\section{References}

1. G. Ferrer and A. Sanfeliu, "Proactive kinodynamic planning using the extended social force model and human motion prediction in urban environments," in IEEE/RSJ international conference on Intelligent robots and systems. IEEE, 2014, pp. 1730-1735.

2. I. Leite, C. Martinho, and A. Paiva, "Social robots for long-term interaction: a survey," International Journal of Social Robotics, vol. 5, no. 2, pp. 291-308, 2013.

3. A. Bauer, D. Wollherr, and M. Buss, "Human-robot collaboration: a survey," International Journal of Humanoid Robotics, vol. 5, no. 01, pp. 47-66, 2008.

4. M. A. Goodrich and A. C. Schultz, "Human-robot interaction: a survey," Foundations and trends in humancomputer interaction, vol. 1, no. 3, pp. 203-275, 2007.

5. T. Kanda, M. Shiomi, Z. Miyashita, H. Ishiguro, and N. Hagita, "An affective guide robot in a shopping mall," in Proceedings of the 4 th ACM/IEEE international conference on Human robot interaction, 2009, pp. 173-180.

6. H.-M. Gross, H. Boehme, C. Schroeter, S. Müller, A. König, E. Einhorn, C. Martin, M. Merten, and A. Bley, "Toomas: interactive shopping guide robots in everyday use-final implementation and experiences from long-term field trials," in IEEE/RSJ International Conference on Intelligent Robots and Systems, 2009, pp. 2005-2012.

7. A. Garrell and A. Sanfeliu, "Cooperative social robots to accompany groups of people," The International Journal of Robotics Research, vol. 31, no. 13, pp. 1675-1701, 2012.
8. M. Kuderer and W. Burgard, "An approach to socially compliant leader following for mobile robots," in International Conference on Social Robotics. Springer, 2014, pp. 239-248.

9. F. Yuan, M. Hanheide, G. Sagerer, et al., "Spatial context-aware person-following for a domestic robot," International Workshop on Cognition for Technical Systems, 2008.

10. V. N. The and C. Jayawardena, "A decision making model for optimizing social relationship for side-by-side robotic wheelchairs in active mode," in 6th IEEE International Conference on Biomedical Robotics and Biomechatronics, 2016, pp. 735-740.

11. A. Rodić, M. Vujović, I. Stevanović, and M. Jovanović, "Development of human-centered social robot with embedded personality for elderly care," in New Trends in Medical and Service Robots. Springer, 2016, pp. 233-247.

12. Y. Morales, T. Kanda, and N. Hagita, "Walking together: side by side walking model for an interacting robot," Journal of Human-Robot Interaction, vol. 3, no. 2, pp. 5173, 2014.

13. D. Carton, A. Turnwald, D. Wollherr, and M. Buss, "Proactively approaching pedestrians with an autonomous mobile robot in urban environments," in Experimental Robotics. Springer, 2013, pp. 199-214.

14. S. Satake, T. Kanda, D. F. Glas, M. Imai, H. Ishiguro, and N. Hagita, "How to approach humans?-strategies for social robots to initiate interaction," in IEEE International Conference on Human-Robot Interaction, 2009, pp. 109-116.

15. F. Zanlungo, T. Ikeda, and T. Kanda, "Potential for the dynamics of pedestrians in a socially interacting group," Physical Review E, vol. 89, no. 1, p. 012811, 2014.

16. T. Ikeda, Y. Chigodo, D. Rea, F. Zanlungo, M. Shiomi, and T. Kanda, "Modeling and prediction of pedestrian behavior based on the sub-goal concept," Robotics: Science and Systems VIII, p. 137, 2013.

17. Y. Chen, F. Wu, W. Shuai, N. Wang, R. Chen, and $\mathrm{X}$. Chen, "Kejia robot-an attractive shopping mall guider," in International Conference on Social Robotics. Springer, 2015, pp. 145-154.

18. W. C. Pang, G. Seet, and X. Yao, "A multimodal personfollowing system for telepresence applications," in Proceedings of the 19th ACM Symposium on Virtual Reality Software and Technology. ACM, 2013, pp. 157-164.

19. H.-M. Gross, C. Schroeter, S. Mueller, M. Volkhardt, E. Einhorn, A. Bley, C. Martin, T. Langner, and M. Merten, "Progress in developing a socially assistive mobile home robot companion for the elderly with mild cognitive impairment," in IEEE/RSJ International Conference on Intelligent Robots and Systems, 2011, pp. 24302437.

20. E. Prassler, D. Bank, and B. Kluge, "Motion coordination between a human and a robotic wheelchair," in Proceedings. 10th IEEE International Workshop on Robot and Human Interactive Communication, 2001, pp. 412-417.

21. Y. Kobayashi, Y. Kinpara, T. Shibusawa, and Y. Kuno, "Robotic wheelchair based on observations of people using integrated sensors," in IEEE/RSJ International Conference on Intelligent Robots and Systems, 2009, pp. 20132018.

22. R. Suzuki, T. Yamada, M. Arai, Y. Sato, Y. Kobayashi, and Y. Kuno, "Multiple robotic wheelchair system considering group communication," in International Symposium on Visual Computing. Springer, 2014, pp. 805-814. 
23. V. K. Narayanan, A. Spalanzani, F. Pasteau, and M. Babel, "On equitably approaching and joining a group of interacting humans," in IEEE/RSJ International Conference on Intelligent Robots and Systems, 2015, pp. 4071-4077.

24. Y. Kato, T. Kanda, and H. Ishiguro, "May i help you?: Design of human-like polite approaching behavior," in Proceedings of the Tenth Annual ACM/IEEE International Conference on Human-Robot Interaction. ACM, 2015, pp. $35-42$.

25. K. Charalampous, I. Kostavelis, and A. Gasteratos, "Recent trends in social aware robot navigation: A survey," Robotics and Autonomous Systems, vol. 93, pp. 85-104, 2017.

26. T. Kruse, A. K. Pandey, R. Alami, and A. Kirsch, "Human-aware robot navigation: A survey," Robotics and Autonomous Systems, vol. 61, no. 12, pp. 1726-1743, 2013.

27. E. Owen and L. Montano, "Motion planning in dynamic environments using the velocity space," in IEEE/RSJ International Conference on Intelligent Robots and Systems. IEEE, 2005, pp. 2833-2838.

28. A. F. Foka and P. E. Trahanias, "Probabilistic autonomous robot navigation in dynamic environments with human motion prediction," International Journal of Social Robotics, vol. 2, no. 1, pp. 79-94, 2010.

29. P. Henry, C. Vollmer, B. Ferris, and D. Fox, "Learning to navigate through crowded environments," in International Conference on Robotics and Automation, 2010, pp. 981-986.

30. P. Trautman, J. Ma, R. M. Murray, and A. Krause, "Robot navigation in dense human crowds: Statistical models and experimental studies of human-robot cooperation," The International Journal of Robotics Research, vol. 34, no. 3, pp. 335-356, 2015.

31. M. Kuderer, H. Kretzschmar, C. Sprunk, and W. Burgard, "Feature-based prediction of trajectories for socially compliant navigation." in Robotics: science and systems, 2012.

32. M. Luber, L. Spinello, J. Silva, and K. O. Arras, "Socially-aware robot navigation: A learning approach," in IEEE/RSJ international conference on Intelligent robots and systems, 2012, pp. 902-907.

33. E. A. Sisbot, L. F. Marin-Urias, R. Alami, and T. Simeon, "A human aware mobile robot motion planner," IEEE Transactions on Robotics, vol. 23, no. 5, pp. 874-883, 2007.

34. A. Ohya, "Human robot interaction in mobile robot applications," in Proceedings. 11th IEEE International Workshop on Robot and Human Interactive Communication, 2002 , pp. $5-10$.

35. J.-S. Hu, J.-J. Wang, and D. M. Ho, "Design of sensing system and anticipative behavior for human following of mobile robots," IEEE Transactions on Industrial Electronics, vol. 61, no. 4, pp. 1916-1927, 2014.

36. K. Nakazawa, K. Takahashi, and M. Kaneko, "Movement control of accompanying robot based on artificial potential field adapted to dynamic environments," Electrical Engineering in Japan, vol. 192, no. 1, pp. 25-35, 2015.

37. L. Y. Morales Saiki, S. Satake, R. Huq, D. Glas, T. Kanda, and N. Hagita, "How do people walk side-byside?: using a computational model of human behavior for a social robot," in Proceedings of the seventh annual ACM/IEEE international conference on Human-Robot Interaction. ACM, 2012, pp. 301-308.

38. R. Murakami, L. Y. Morales Saiki, S. Satake, T. Kanda, and H. Ishiguro, "Destination unknown: walking sideby-side without knowing the goal," in Proceedings of the ACM/IEEE international conference on Human-robot interaction. ACM, 2014, pp. 471-478.
39. D. Karunarathne, Y. Morales, T. Kanda, and H. Ishiguro, "Model of side-by-side walking without the robot knowing the goal," International Journal of Social Robotics, vol. 10, no. 4, pp. 401-420, 2018.

40. M. Kuderer, H. Kretzschmar, and W. Burgard, "Teaching mobile robots to cooperatively navigate in populated environments," in IEEE/RSJ International Conference on Intelligent Robots and Systems. IEEE, 2013, pp. 31383143.

41. E. T. Hall and T. Edward, "Hall. the hidden dimension," Anchor Books New York, vol. 20, p. 71, 1969.

42. M. L. Walters, K. Dautenhahn, R. Te Boekhorst, K. L. Koay, C. Kaouri, S. Woods, C. Nehaniv, D. Lee, and I. Werry, "The influence of subjects' personality traits on personal spatial zones in a human-robot interaction experiment," in IEEE International Workshop on Robot and Human Interactive Communication, 2005, pp. 347-352.

43. D. S. Syrdal, K. L. Koay, M. L. Walters, and K. Dautenhahn, "A personalized robot companion?-the role of individual differences on spatial preferences in hri scenarios," in The 16th IEEE International Symposium on Robot and Human interactive Communication, 2007, pp. 1143-1148.

44. S. Woods, M. Walters, K. L. Koay, and K. Dautenhahn, "Comparing human robot interaction scenarios using live and video based methods: towards a novel methodological approach," in 9th IEEE International Workshop on Advanced Motion Control, 2006, pp. 750-755.

45. F. Belkhouche, B. Belkhouche, and P. Rastgoufard, "Line of sight robot navigation toward a moving goal," IEEE Transactions on Systems, Man, and Cybernetics, Part B (Cybernetics), vol. 36, no. 2, pp. 255-267, 2006.

46. L. Freda and G. Oriolo, "Vision-based interception of a moving target with a nonholonomic mobile robot," Robotics and Autonomous Systems, vol. 55, no. 6, pp. 419432, 2007.

47. I. R. Manchester, E.-M. Low, and A. V. Savkin, "Interception of a moving object with a specified approach angle by a wheeled robot: Theory and experiment," in 47 th IEEE Conference on Decision and Control, 2008, pp. 490495.

48. B. R. Fajen and W. H. Warren, "Behavioral dynamics of intercepting a moving target," Experimental Brain Research, vol. 180, no. 2, pp. 303-319, 2007.

49. E. Avrunin and R. Simmons, "Using human approach paths to improve social navigation," in 8th ACM/IEEE International Conference on Human-Robot Interaction, 2013, pp. 73-74.

50. A. Garrell, M. Villamizar, F. Moreno-Noguer, and A. Sanfeliu, "Teaching robots proactive behavior using human assistance," International Journal of Social Robotics, vol. 2, no. 9, pp. 231-249, 2017.

51. D. Carton, W. Olszowy, D. Wollherr, and M. Buss, "Socio-contextual constraints for human approach with a mobile robot," International Journal of Social Robotics, vol. 9, no. 2, pp. 309-327, 2017.

52. G. Ferrer and A. Sanfeliu, "Bayesian human motion intentionality prediction in urban environments," Pattern Recognition Letters, vol. 44, pp. 134-140, 2014.

53. G. Ferrer, A. Garrell, F. Herrero, and A. Sanfeliu, "Robot social-aware navigation framework to accompany people walking side-by-side," Autonomous Robots, pp. 1-19, 2016.

54. G. Ferrer and A. Sanfeliu, "Multi-objective cost-to-go functions on robot navigation in dynamic environments," in IEEE/RSJ international conference on Intelligent robots and systems. IEEE, 2015, pp. 3824-3829. 
55. E. Repiso, G. Ferrer, and A. Sanfeliu, "On-line adaptive side-by-side human robot companion in dynamic urban environments," in IEEE/RSJ international conference on Intelligent robots and systems, 2017, pp. 3824-3829.

56. D. Katagami and S. Yamada, "Active teaching for an interactive learning robot," in Proceedings on the 12th Robot and Human Interactive Communication, 2003, pp. 181-186.

57. E. Repiso, A. Garrell, and A. Sanfeliu, "Robot approaching and engaging people in a human-robot companion framework," in 2018 IEEE/RSJ International Conference on Intelligent Robots and Systems (IROS). IEEE, 2018, pp. 8200-8205.

58. V. K. Narayanan, A. Spalanzani, R. C. Luo, and M. Babel, "Analysis of an adaptive strategy for equitably approaching and joining human interactions," in 25th IEEE International Symposium on Robot and Human Interactive Communication, 2016, pp. 341-346.

59. D. Helbing and P. Molnar, "Social force model for pedestrian dynamics," Physical review E, vol. 51, no. 5, p. 4282, 1995.
60. F. Zanlungo, T. Ikeda, and T. Kanda, "Social force model with explicit collision prediction," EPL (Europhysics Letters), vol. 93, no. 6, p. 68005, 2011.

61. D. E. Goldberg and J. H. Holland, "Genetic algorithms and machine learning," Machine learning, vol. 3, no. 2, pp. 95-99, 1988.

62. K. Stubbs, P. J. Hinds, and D. Wettergreen, "Autonomy and common ground in human-robot interaction: A field study," IEEE Intelligent Systems, vol. 22, no. 2, 2007.

63. E. Trulls, A. Corominas Murtra, J. Pérez-Ibarz, G. Ferrer, D. Vasquez, J. M. Mirats-Tur, and A. Sanfeliu, "Autonomous navigation for mobile service robots in urban pedestrian environments," Journal of Field Robotics, vol. 28, no. 3, pp. 329-354, 2011.

64. V. Vaquero, E. Repiso, and A. Sanfeliu, "Robust and real-time detection and tracking of moving objects with minimum 2d lidar information to advance autonomous cargo handling in ports," Sensors, vol. 19, no. 1, p. 107, 2019 . 